\title{
Velocity extension for the Level-set method and multiple eigenvalues in shape optimization
}

\author{
Frédéric de Gournay *
}

September 28, 2005

\begin{abstract}
In the context of structural optimization by the level-set method, we propose an extension of the velocity of the underlying Hamilton-Jacobi equation. The gradient method is endowed with an Hilbertian structure based on the $H^{1}$ Sobolev space. Numerical results for compliance minimization and mechanism design shows a strong improvement of the rate of convergence of the level-set method. Another important application is the optimization of multiple eigenvalues.
\end{abstract}

Keywords: multiple eigenvalues, shape optimization, sensitivity analysis, shape derivative, level-set method, regularization

*Centre de Mathématiques Appliquées (UMR 7641), Ecole Polytechnique, 91128 Palaiseau, France - degourna@cmapx . polytechnique.fr 


\section{Introduction}

Optimal design of elastic structures has been widely studied and many different numerical methods are used to solve this problem. Most of existing methods can be divided into two main classes : Topology optimization which optimizes a density of material in each cell and Geometric optimization which moves the boundary of the domain.

The most recent topology method, the homogeneization method and its variants (power-law method or SIMP method) may be considered quite classical in view of the number of publications (see e.g. [All01], [ABFJ97], [Ben95], [BS03], [BK88], [Che00]). The homogeneization method seems the most promising because it is independent with respect to initialization and because it gives strong mathematical result of existence of solution. Sadly this method bears the difficulty of handling quite badly eigenvalue problems where apparitions of so-called fictitious-material modes prevents stable numerical computation. Another problem is that topology methods give optimal shapes that are composite. Penalization methods allow to project the composite shape on a classical shape (a black-and-white design).

The problem of fictitious modes is naturally solved with the geometric methods where the shape is clearly identified and the void cannot generate fictitious modes.

The major drawback of geometric methods is their dependency with respect to initialization. Even the most recent level set method ([AJ05],[AJT04], [WWG03]) is very sensitive to initialization although the topology can change. In order to avoid this problem, a method of topology optimization, the bubble method, (or topological gradient method [ES94], [GGM01], [SJP92] [SZ01]) has been recently coupled with geometric optimization ([AGJT05], [BHR04]). Another recent advance in that field has been recently led by [NS04], but numerical use of this method is still undone.

We wish here to correct the so-called void problem that arises when using the level set algorithm. This problem is generated by the weak material approximation that give rise to a negative velocity for advecting the shape in the void. The void problem slows down the algorithm when mesh is refined.

The void problem is presented in Section $\mathbf{3}$ and the chosen solution is presented in Section 4. The method mainly consists in applying the $\Delta^{-1}$ operator to the velocity which is costless. This method allows to regularize, extend and gives a local Hilbertian structure (see discussion of Section 4.2). Numerical results are presented in Section $\mathbf{4 . 3}$ to $\mathbf{4 . 7}$, they compare the new method with the previous one and show strong improvements of the level set algorithm.

It also has been made possible to deal with the problem of optimizing an eigenvalue when its multiplicity is greater than one. Theoretical differentiation of the eigenvalue is made in Section 5. As can be expected from theory on eigenvalue differentiation, the first eigenvalue is directionally differentiable with respect to shape variation, even when this eigenvalue is multiple. The algorithm that optimizes the first eigenvalue is detailed in Section 5.3. It strongly relies 
on the local Hilbertian structure given by velocity regularization. Indeed, because of this local Hilbertian structure, the differential gives rize to a gradient and therefore to a steepest descent. We show that the choice of the steepest descent is a Semi-Definite Program in low dimension that is easily solved. Some numerical results are presented in Section 6.

The method used for optimizing multiple eigenvalues can be extended to other criteria that are not differentiable as the robust compliance criterion in the sense of [CC03]. The method of velocity regularization presented here is an adaptation of [Bur03], [PBH04], [MP01]. It is a standard issue in numerical computation.

\section{The level-set method in shape optimization}

\subsection{Eigenvalue maximization}

We set our model problem in linearized elasticity. Let $\Omega \subset \mathbb{R}^{d}(d=2$ or 3 ) be a bounded open set occupied by a linear isotropic elastic material with Hooke's law $A_{0}$. Recall that, for any symmetric matrix $\xi, A_{0}$ is defined by

$$
A_{0} \xi=2 \mu \xi+\lambda(\operatorname{Tr} \xi) I d,
$$

where $\mu$ and $\lambda$ are the Lamé coefficients of the material. The boundary of $\Omega$ is made of two disjoint parts

$$
\partial \Omega=\Gamma_{N} \cup \Gamma_{D}
$$

with Dirichlet boundary conditions on $\Gamma_{D}$, and Neumann boundary conditions on $\Gamma_{N}$. The spectral problem is to find an eigenvalue $\gamma$ and an eigenvector $u \neq 0$ such that:

$$
\left\{\begin{aligned}
-\operatorname{div}(A e(u)) & =\gamma \rho u & & \text { in } \Omega \\
u & =0 & & \text { on } \Gamma_{D} \\
(A e(u)) n & =0 & & \text { on } \Gamma_{N}
\end{aligned}\right.
$$

Where $\rho$ is a scalar field on $\Omega$ that represents the material density (typically $\rho$ is equal to 1 on $\Omega$ ).

It is well known that $S p(\Omega)$ the set of eigenvalues is a countable set of positive numbers that tends to infinity. The smallest eigenvalue $\gamma_{1}(\Omega)=\min S p(\Omega)$ can then be defined.

A classical way of improving the rigidity of a structure is to maximize the first eigenfrequency. Thus, a natural objective function to be minimized is

$$
\mathcal{L}(\Omega)=-\gamma_{1}(\Omega)+\eta|\Omega| .
$$

Where $\eta$ is a given Lagrange multiplier for a volume constraint. We want to minimize $\mathcal{L}$ with respect to $\Omega$ with a constraint that $\Omega \subset D$ where $D$ is a given domain of $\mathbb{R}^{d}$. 


\subsection{Classical algorithm}

The works [OS01], [SW00] or [AJT04] give an extensive explanation of the levelset method. Only the main ideas are to be reminded in this Section.

As described in [AJ05], when the dimension of the eigenspace associated to $\gamma_{1}$ is equal to 1 , the above functional $\mathcal{L}(\Omega)$ is differentiable with respect to variation of the domain and the geometrical shape optimization method can be applied. It reads :

- Calculation of the gradient Let $\Omega_{k}$ be the domain at iteration $k$. Assuming that $\gamma_{1}\left(\Omega_{k}\right)$ is simple, for a given $\theta \in W^{1, \infty}(D ; D)$ define

$$
(I d+\theta) \circ \Omega_{k}=\left\{x+\theta(x) \text { with } x \in \Omega_{k}\right\} .
$$

Prove that $\mathcal{L}\left((I d+\theta) \circ \Omega_{k}\right)$ is differentiable with respect to $\theta$ at the point $\theta=0$. The value of $\mathcal{L}^{\prime}$, the differential at the point 0 is given by :

$$
\begin{aligned}
\mathcal{L}^{\prime}(\theta)= & \int_{\partial \Omega_{k}}(\theta \cdot n)(-v+\eta) \\
\text { With } & \left\{\begin{array}{lll}
v=A e(u): e(u)-\gamma_{1} \rho u \cdot u & \text { on } \Gamma_{N}^{k} \\
v=-A e(u): e(u) & \text { on } \Gamma_{D}^{k}
\end{array} .\right.
\end{aligned}
$$

Where $u$ is an eigenvector normalized by $\int_{\Omega_{k}} \rho u \cdot u=1$ which is, up to a change of sign, by assumption, unique. And where $n$ is the outer normal of $\Omega_{k}$.

- Calculation of a descent direction Choose $\theta_{k}$ such that $\mathcal{L}^{\prime}\left(\theta_{k}\right)<0$ and let $\Omega_{k+1}=\left(I d+t \theta_{k}\right) \circ \Omega_{k}$, where $t$ is the step of the gradient method.

The level set method is a geometrical shape optimization method where the domains $\Omega_{k}$ are represented through functions $\phi_{k}$ defined on $D$ in the following way: $x \in \Omega_{k} \Longleftrightarrow \phi_{k}(x)<0$. Such a $\phi_{k}$ is said to be "a level set function of $\Omega_{k}$ ". Of course for a given domain, the associated level set functions are not unique.

The changes of topology are then handled in a very simple way (see [AJT04] for extensive explanations of numerical advantages). The method relies on the following lemma :

Lemma 2.1 If $\phi_{k}$ is a level set function of $\Omega_{k}$, define $\phi_{k+1}$ as :

$$
\phi_{k+1}-\phi_{k}+t\left(\theta_{k} \cdot n\right)\left|\nabla \phi_{k}\right|=0 .
$$

Then there exist an $O\left(t^{2}\right)$ such that $\phi_{k+1}+O\left(t^{2}\right)$ is a level set function for the domain $\Omega_{k+1}=\left(I d+t \theta_{k}\right) \circ \Omega_{k}$

Thus, the following scheme for choosing $\phi_{k+1}$ is the most commonly used

$$
\begin{aligned}
\phi(0) & =\phi_{k} \\
\frac{\partial \phi}{\partial t}+V^{*}|\nabla \phi| & =0 \\
\phi_{k+1} & =\phi(T)
\end{aligned}
$$


where $T>0$ is the step of the gradient method and $V^{*}$ is the descent direction chosen accordingly to the calculation (4) of the differential of $J$. Defining $V^{*}=$ $v-\eta(v$ being defined in (4)) is the most commonly used choice, we will call this choice the natural extension method. The goal of this paper is to find a different way of defining $V^{*}$.

Remark 2.2 In order to avoid multiple definition of $V^{*}$ in the Natural extension method, it is supposed that the Dirichlet part of the boundary is fixed and that the $v$ used everywhere in the domain is the one defined for $\Gamma_{N}$.

\section{The weak material approximation and the nat- ural extension method}

The stiffness matrix that corresponds to the linear operator of elasticity is equal to zero on the nodes that does not belong to $\Omega$ i.e.on $x \in D$ such that $\phi(x)>$ 0 .Then this matrix is not invertible. In order to avoid this problem, the so-called weak material (or ersatz material) approximation consists in fixing Hooke's law $A$ and material density $\rho$ as :

$$
\begin{aligned}
& A=A_{0} \text { and } \rho=1 \text { in } \Omega \\
& A=\varepsilon A_{0} \text { and } \rho=\varepsilon^{\alpha} \quad \text { outside } \Omega
\end{aligned}
$$

with a small parameter $\varepsilon$ and $\alpha \geq 1$. The fictitious modes are avoided by setting $\alpha=\infty$. In the continuous case, it has been proven (see [SHSP89]) that the eigenvector computed with the ersatz material approximation is equal at first order in $\varepsilon$ to the desired eigenvector introduced in (2).

Recalling that the Dirichlet part of the boundary of $\partial \Omega$ has been fixed ( see remark 2.2), $v-\eta=A e(u): e(u)-\gamma_{1} \rho u \cdot u-\eta$ is now defined everywhere on $D$ and the natural extension method consists in defining $V^{*}=A e(u): e(u)-\gamma_{1} \rho u \cdot u-\eta$ on every cell.

This nevertheless raises a problem : since $u$ is everywhere of order $\varepsilon^{0}$, then $A e(u): e(u)$ is of order $\varepsilon$ outside $\Omega$ which means that the velocity extension is almost equal to $-\eta$ outside the domain, and makes it very difficult for the shape to increase its volume. Even if the descent step $T$ is increased in order to speed up the method, the parts of the shape where there is a need to decrease the volume will move faster than the parts of the shape where there is a demand on increasing the volume.

Numerical practice shows that between each computation of the eigenvalue, the level-set method can not move $\partial \Omega$ of more than one cell away from the original boundary when it wants to improve the volume. This leads to a drastically increasing computational time with mesh-refinement.

This remark is true, of course, for every objective function and not only for the minimization of the first eigenvalue. 


\section{Velocity regularization by the Hilbertian method}

\subsection{Definition of the Hilbertian method}

We will suppose that the domain $\Omega$ has enough regularity so that $v(\cdot, \cdot)$ defined in (4) belongs to $H^{-1 / 2}(\partial \Omega)$. Even if the optimal domain may possibly be irregular, physical and numerical intelligence tells that throughout optimization $v(\cdot, \cdot)$ has the required regularity but mathematical proof of this fact is still lacking. Let us first define a scalar product :

Definition 4.1 For $a \in \mathbb{R}^{*+}$, define the following scalar product on $H^{1}(D)$

$$
(u, w)_{H^{1}}=\int_{D} a \nabla u \cdot \nabla w+u w
$$

With the associated norm $\|\cdot\|_{H^{1}}$.

The velocity $V^{*}$ in the Hamilton-Jacobi equation (6) is chosen as the unique solution to

$$
\int_{\partial \Omega} V^{*}(-v+\eta)=\min _{\substack{V \in H^{1}(D) \\\|V\|_{H^{1}}=1}} \int_{\partial \Omega} V(-v+\eta)
$$

Where $v$ is the differential defined in (5) and $\Omega$ is the actual working domain.

\subsection{Advantages of the method}

$\diamond$ Scalar versus vector: We choose to extend the normal velocity which is a scalar field, i.e.: If $\theta$ is the vector field that advects the domain, its normal velocity is equal to $\theta \cdot n$ on the boundary of the domain. Seen as a distribution over $D$, the normal velocity is more regular than the vector velocity. A good example is when two parts of the boundary want to merge : $\theta \cdot n$ is positive on the two parts and $\theta$ changes orientation. This situation gets worst at the next step of the algorithm (see Figure 1).

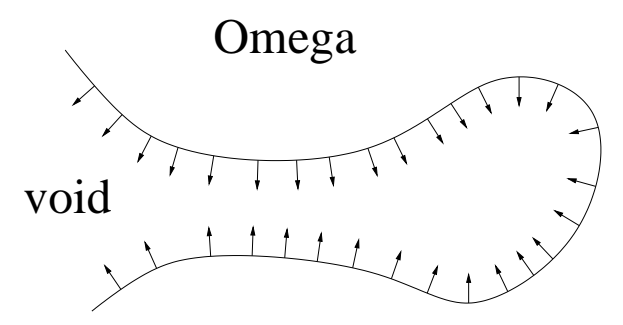

Figure 1: Two merging parts of the boundary leads to a non-regular vector field as seen as a distribution on $D$. 
$\diamond$ How to compute $V^{*}$ : The problem of finding $V^{*}$ is not difficult: Let $\bar{V}$ be the unique solution to

$$
(\bar{V}, X)_{H^{1}}=\int_{\partial \Omega} X(-v+\eta) \quad \forall X \in H^{1}(D)
$$

Where $(\cdot, \cdot)_{H^{1}}$ is the scalar product of definition 4.1. Then $V^{*}=-\frac{\bar{V}}{\|\bar{V}\|_{H^{1}}}$ and the inversion of the matrix that corresponds to the scalar product $(\cdot, \cdot)_{H^{1}}$ has to be done only once in the optimization process.

$\diamond$ Extension, Regularization and Hilbertian structure: Three different goals are sought in the Hilbertian extension of the velocity.

First, the formula for the differential of $\mathcal{L}$ gives a velocity that makes sense only on the boundary of the domain and the Hamilton-Jacobi equation (6) needs a speed defined everywhere on the domain or else the algorithm cannot move the boundary of more than one cell during optimization, this is the extension issue.

Secondly, the velocity is regularized by the application of a $\Delta^{-1}$ operator. This is the regularization issue. It is expected to increase the accuracy of the algorithm and is a standard issue in optimization problems (see e.g. [PBH04], [Bur03],[MP01]).

Thirdly, the problem is endowed with an Hilbertian structure and we work with gradient-type methods, this issue will be developed later in Section 5 .

$\diamond$ Hilbertian extension versus other extensions: The most natural way to extend the velocity outside $\partial \Omega$ would have been to extend $v-\eta$ according to the normal of $\partial \Omega$ by a front-propagating type method as the fast-marching method described in [OS88]. This method do not endow the space with an Hilbertian structure. Neither does it regularize the velocity.

$\diamond V^{*}$ is indeed an extension: Because the scalar product diffuses the source term, the velocity is now defined everywhere on $D$ and the typical problem of null velocity in the void that raises with the natural extension method is now cured.

$\diamond V^{*}$ is indeed a regularization: The speed used in the Hilbertian extension method, $V^{*}$ is more regular than $v-\eta$ (the speed used in the Natural extension method) as can be seen in the following formal derivation :

If $v$ belongs to an $H^{s}(D)$ then $X \mapsto \int_{\partial \Omega} X(-v+\eta)$ is a linear form on $H^{-s+1 / 2}(D)$ and $V^{*}$ belongs to an $H^{s+3 / 2}(D)$ by elliptic regularity on the very smooth domain $D$. Indeed $V^{*}$ is obtained by operating an inverse Laplacian on the distribution (on $D) X \mapsto \int_{\partial \Omega} X(-v+\eta)$ and so gains two derivatives.

$\diamond$ Consistency of the extension: This algorithm could be seen as a gradient-type algorithm if there were a vector field $\theta^{*} \in W^{1, \infty}(D ; D)$ such that $\theta^{*} \cdot n=V^{*}$ on $\partial \Omega$. Two hypothesis would then be needed: a certain regularity on $V^{*}$ and a certain regularity of the domain $\Omega$ itself.

In order to ensure regularity of $V^{*}$, a scalar product on $H^{p}, p \geq 1$ could have been used instead of a scalar product on $H^{1}$. In this case, formally, $V^{*}$ would have $2 p-1 / 2$ more derivatives in $L^{2}$ than $v$. But numerical computation of 
$V^{*}$ may be quite difficult. Indeed, the computation of the matrix of the scalar product of $H^{p}$ needs finite elements that are chosen accordingly to $p$.

$\diamond$ The extension parameters: The coefficient $a$ characterize the diffusion of $v-\eta$ in the sense that setting it small compared to 1 will lead to a solution $V^{*}$ which is pointwise almost equal to $v-\eta$ on $\partial \Omega$ on equal to zero outside $\partial \Omega$. It must be set small enough so that a big value of $|v-\eta|$ on one part of the boundary does not interfere too much with the values of $v-\eta$ on the other parts of the boundary. But it must be set big enough in order to diffuse the value of $v-\eta$ outside the boundary of $\Omega$.

\subsection{Numerical example: Eigenvalues of a cantilever}

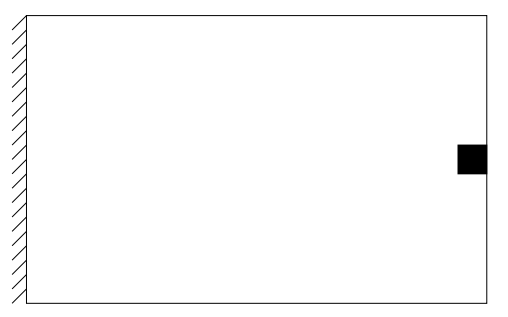

Figure 2: Boundary conditions for a 2-d cantilever (the black zone is heavier and not subject to optimization).

We study a medium-cantilever problem. The working domain is a $2 \times 1$ rectangle, with zero displacement condition on the left side and a small square region at the middle of the right side (see Figure 2) which is 500 times heavier and not subject to optimization. This heavier mass allows to avoid trivial shapes. The Young modulus of the material is set to 1 , the Poisson ratio $\nu$ to 0.3 and the Lagrange multiplier to $7 \times 10^{-2}$. In the void, the ersatz material has a Young modulus set to $10^{-5}$ and a density $\rho$ to 0 . The mesh is refined alternatively in each direction by a factor 2 and the number of transport iteration is adequately increased at each mesh refinement (see Table 1 below).
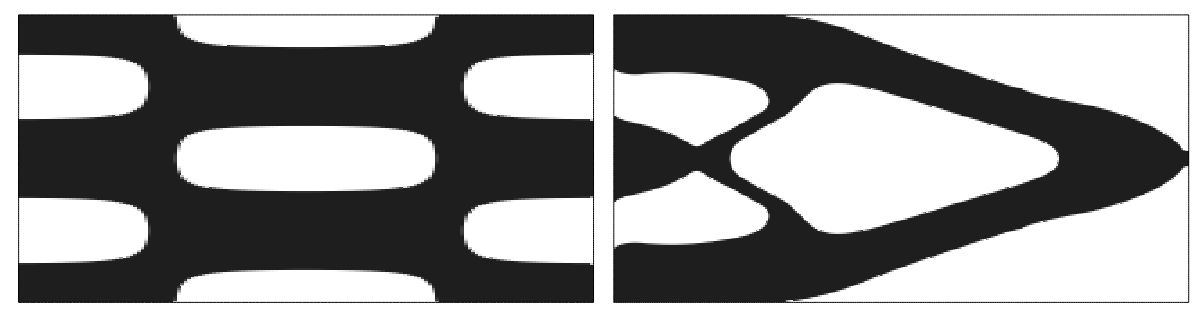

Figure 3: Initialization and optimal shape for the first eigenvalue of a cantilever.

$\diamond$ Explanation of Table 1: That table lists, for different meshes, the number of transport iteration used for each optimization step in the Hamilton- 


\begin{tabular}{|c|c|ccc|ccc|}
\hline \multirow{2}{*}{ Mesh } & $\begin{array}{c}N^{o} \text { of } \\
\text { Transport }\end{array}$ & \multicolumn{2}{|c|}{$\begin{array}{c}\text { Time per iteration } \\
\text { Hilbertian / Natural }\end{array}$} & \multicolumn{3}{|c|}{$\begin{array}{c}\text { Global time } \\
\text { Hilbertian / Natural }\end{array}$} \\
\hline $81 \times 41$ & 16 & 1.46 & $/$ & 1.62 & 30.68 & $/$ & 50.13 \\
$161 \times 81$ & 32 & 9.35 & $/$ & 11.37 & 233.87 & $/$ & 330.01 \\
$321 \times 161$ & 64 & 72.17 & $/$ & 94.91 & 2237.2 & $/$ & 2657.63 \\
\hline $41 \times 41$ & 14 & 0.74 & $/$ & 0.84 & 13.26 & $/$ & 22.6 \\
$81 \times 81$ & 28 & 4.46 & $/$ & 6.19 & 120.62 & $/$ & 136.1 \\
$161 \times 161$ & 56 & 38.50 & $/$ & 46.61 & 1116.57 & $/$ & 1771.44 \\
\hline
\end{tabular}

Table 1: Datas of the optimization of the first eigenvalue of the cantilever.

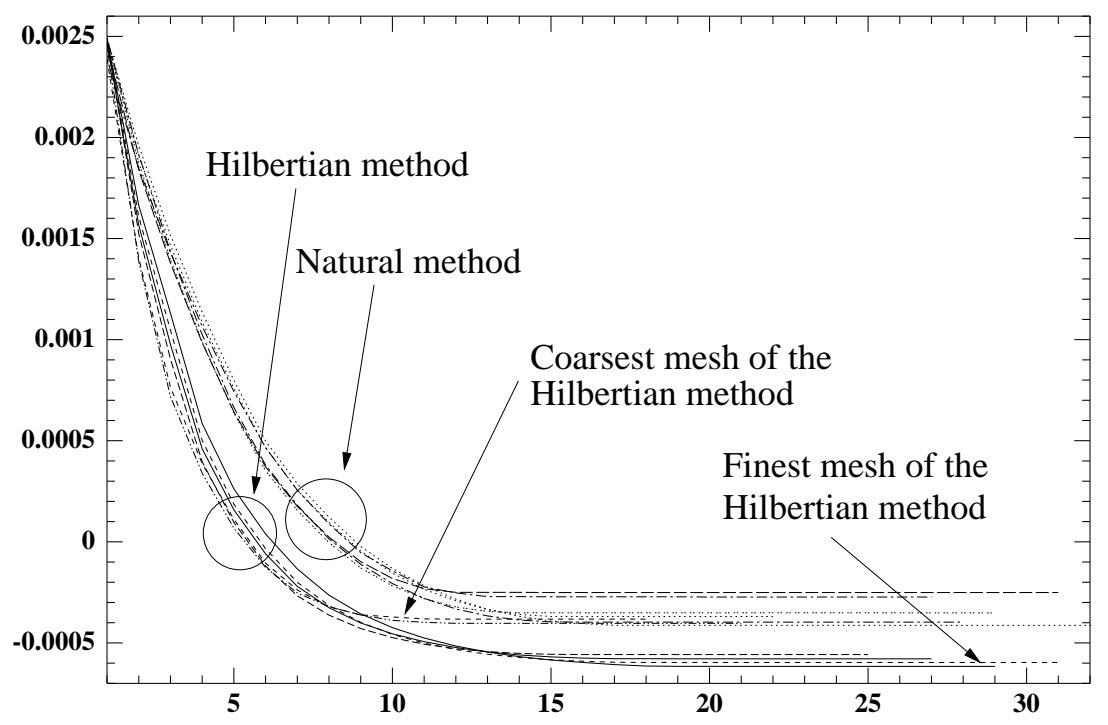

Figure 4: Mesh-refinement influence on the velocity for the Natural extension (left) and the Hilbertian extension (right).

Jacobi equation, the average computed time used per iteration step of the gradient method and the global computing time. The number of transport iteration is shown for the Hilbertian method only. The number of transport iterations for the natural method is 16 times the number of transport iterations of the Hilbertian method. This explains that the average time per iteration of the gradient algorithm is bigger for the Natural method than for the Hilbertian. The latest column is the global computing time for obtaining the optimal shape. The Hilbertian method takes the same amount of time than the Natural method to obtain the optimal shape, but only because it is more accurate: As can be seen on Figure 4, the convergence curves of the Hilbertian method are indeed better than the convergence curves of the Natural method. Time is given in 
seconds.

$\diamond$ Explanation of Figure 4 There is two sets of curves in Figure 4 . The better one are obtained with the Hilbertian method. It appears that the more the mesh is refined, the better the optimal shape is, even if, when mesh is refined, the decreasing of the smallest eigenvalue leads to an increase of the criterion. This is easily explained by a better accuracy on the optimal shape itself.

Remark 4.2 In order to prove mesh-consistency of the algorithm and to have comparable curves in Figure 4, the optimal shape have to be the same when the mesh is changed. Thus those examples are chosen so that there is no possibility for the algorithm to create thin structures when the mesh is refined. This explains why there is a very few holes in the initialization. It is well known that the level-set algorithm can produce more complicated structures.

\subsection{Numerical example : The compliance of the cantilever}
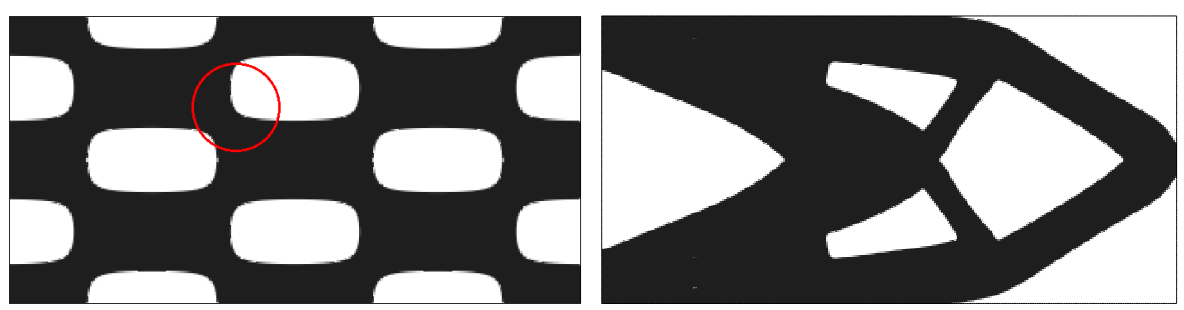

Figure 5: Initialization and optimal shape of the cantilever.

We performed our new velocity extension method on the well-known cantilever problem which is fixed on the left wall an supports a unit vertical point load in the middle of the right wall. The compliance is here optimized. The working domain size is $2 \times 1$. The Young modulus is set to 1 and the Poisson ratio to 0.3 . The Lagrange multiplier is set to $\eta=100$.

Optimization is performed for several finer meshes and the number of transport iteration is multiplied by 2 as each square of the mesh is cut into 4 squares. For the finest mesh $(321 \times 161)$ that corresponds to 51200 elements, the number of transport iterations is equal to 128 for the Natural extension and 16 for the Hilbertian. As a result, the Hilbertian extension is really quicker.

$\diamond$ Mesh-refinement influence: The curves of Figure 6 show that the Hilbertian method is less sensitive to mesh-refinement than the Natural method. Because there is parts of the boundary that have to increase the volume (one of them is encircled in Figure 5) and that the Natural extension method have problems to improve these parts as was said in Section 3, the Natural extension method is sensitive to mesh-refinement. In Figure 4 there was no such demand on improving the volume and the mesh-independence of the Hilbertian method was less obvious. 

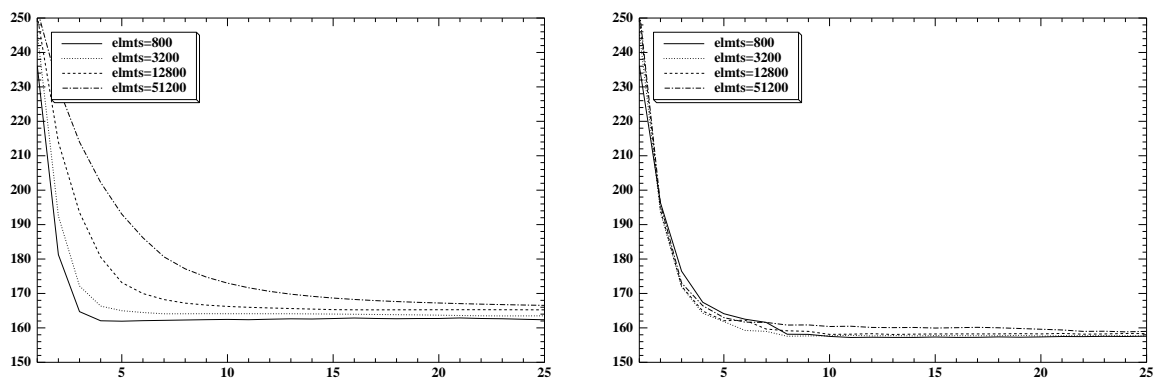

Figure 6: Mesh-refinement influence on the natural extension (left) and the Hilbertian extension (right).

\subsection{Numerical example : A 2-d gripping-mechanism}
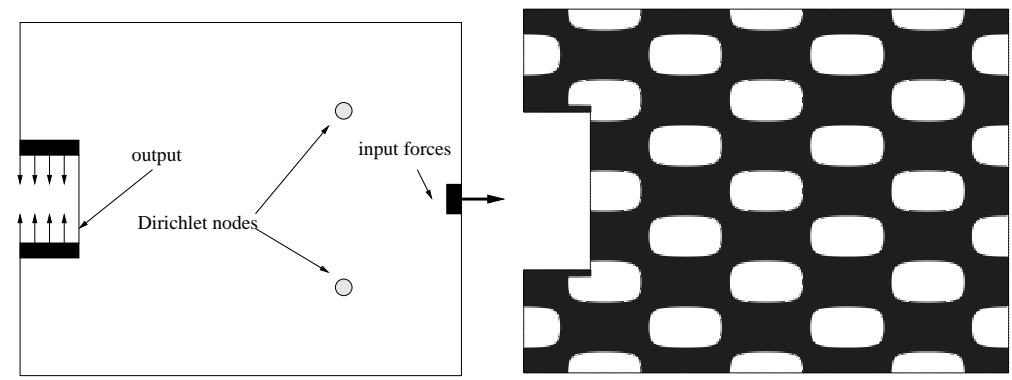

Figure 7: The definition of the 2-d gripping mechanism and its initialization.
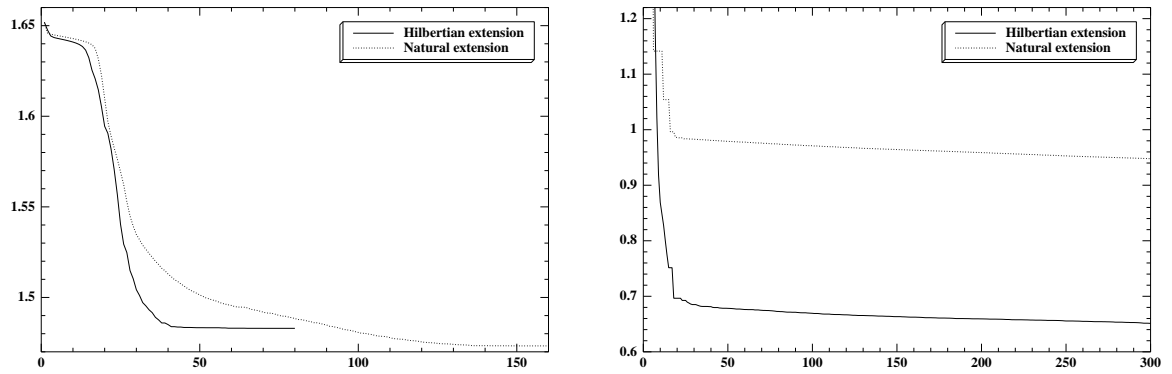

Figure 8: Evolution of the objective function for the first step (left) with a ratio for the weak material equal to 0.01 and the second step with a ratio of $10^{-5}$ (right).

On a $1.2 \times 3.2$ rectangle meshed with $241 \times 641$ nodes, we give a numerical 

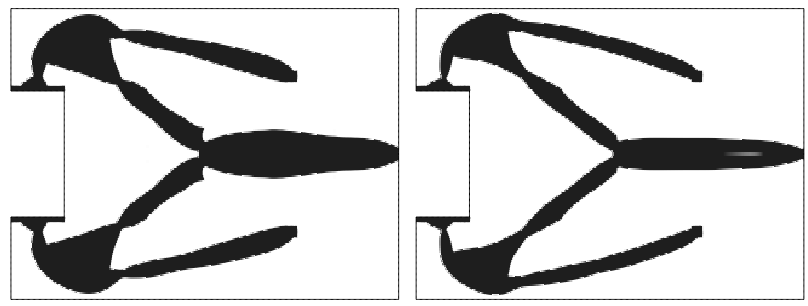

Figure 9: Optimal shape for the Hilbertian method (left) and the natural method (right) at the end of the first step of optimization.
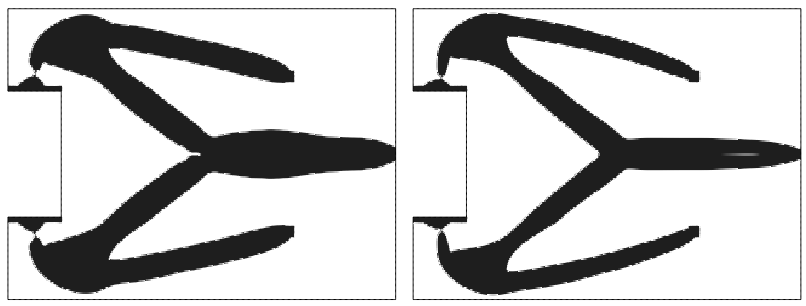

Figure 10: Optimal shape for the Hilbertian method (left) and the natural method (right) at the end of the second step of optimization.

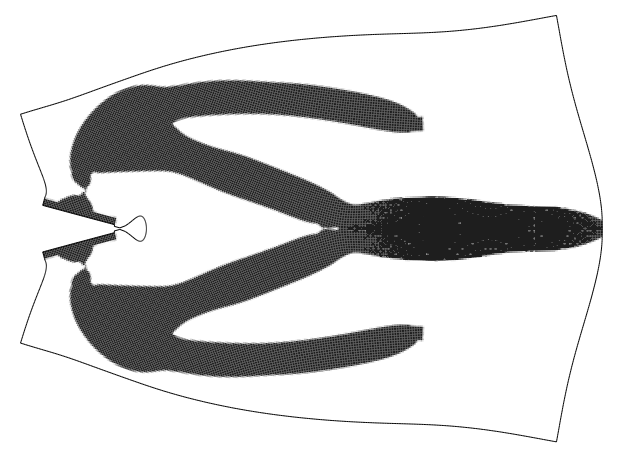

Figure 11: Optimal shape displacement.

example for minimizing the least square of a prescribed displacement. This example was given by the Comissariat à l'Energie Atomique for the design of a grip (see [Ber04]). The objective function is

$$
\mathcal{J}_{l s e}(\Omega)=\int_{\Omega} k(x)\left|u(x)-u_{0}(x)\right|^{2}
$$

where $u$ is the displacement obtained by a given set of forces, $u_{0}$ is a prescribed given displacement and $k$ is a scalar field that characterizes the zone of interest.

The ponderation $k$ is equal to 10 on the left black box of Figure 7 (left); is 
equal to 1 on the right black box and is equal to 0 elsewhere. The prescribed displacement $u_{0}$ is equal to $(0, \pm 1)$ on the left black box and $(0,0)$ on the right box. Enforcing $u$ to be close to zero where the force is input allows to ensure the stiffness and the connectivity of the structure. These black boxes are not subject to optimization.

A force of modulus $1 \mathrm{~N}$ is applied in the $\mathrm{x}$-direction on the middle of the left and a uniform pressure which represents a total force of modulus $5.10^{-2} \mathrm{~N}$ is applied between the jaws of the mechanism in order to enforce the mechanism to hold objects. The prescribed displacement is located on the black box on the left and shown in Figure 7. The Young modulus is set to 1, the Poisson ratio to 0.3 and there is a small Lagrange multiplier of a volume constraint of value 0.05. This Lagrange multiplier helps removing parts of the boundary that are useless.

This is a typical problem where an adjoint is needed. For some incompletely understood reasons, the ratio of the weak material (the factor $\varepsilon$ in Section 3) cannot be set too low (typically it must be at least 1 percent of the strong material) or the algorithm will not work. One of the explanations that may be given is the tendency of the shape to create hinges. The algorithm then concentrate on the hinges only, ignoring the rest of the shape and it is believed it is then stuck in a local minimum. If the ratio of the weak material is high, hinges are less efficient and the previous problem is avoided.

Optimization must then be made in two steps. First the shape is optimized with a ratio of the weak material equal to 1 percent. The optimal shape is then reoptimized with a smaller ratio $\left(10^{-5}\right.$ in this numerical case). In the second optimization procedure, the displacement and the adjoint state are calculated with a more accurate precision which leads to a better precision of the shapederivative.

In Figure 8 (left), it may seem that the Hilbertian method is less efficient than the Natural method. This is explained by the lack of precision in the computation of the shape derivative in the first step of the algorithm. But it is explained too by the lack of precision of the computation of the criterion during the first step of the algorithm. Indeed when the two shapes of Figure 9 (obtained with $\varepsilon=10^{-2}$ ) are computed using $\varepsilon=10^{-5}$, the Hilbertian shape is the better one.

Remark 4.3 The optimal shape for the natural extension method has less volume than the one for the Hilbertian extension, it is a numerical validation of the problem raised in Section 3.

\subsection{Numerical example : More 2-d mechanism}

We briefly present here some more 2-d mechanism, namely a negative Poisson modulus cell (Figure 12 to 14) and a force inverter (Figure 15 to 17). These examples are standards of shape optimization problems and their description can be found in [BS03]. In order to ensure stiffness of the structures, a small pressure load is applied where the displacement is optimized (on the right black 


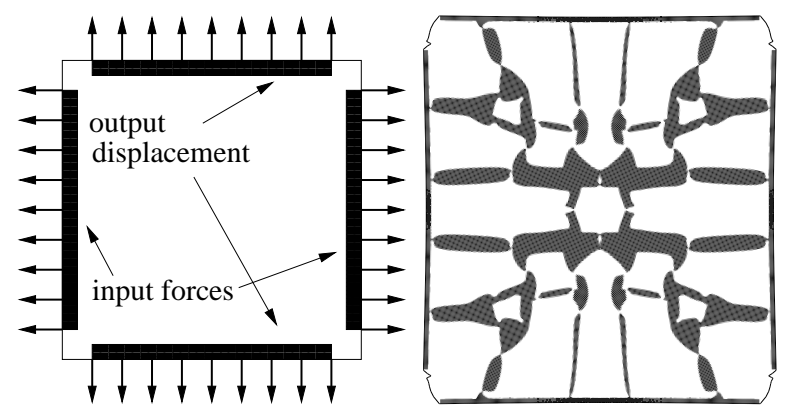

Figure 12: The Negative Poisson modulus problem and its deformed solution.
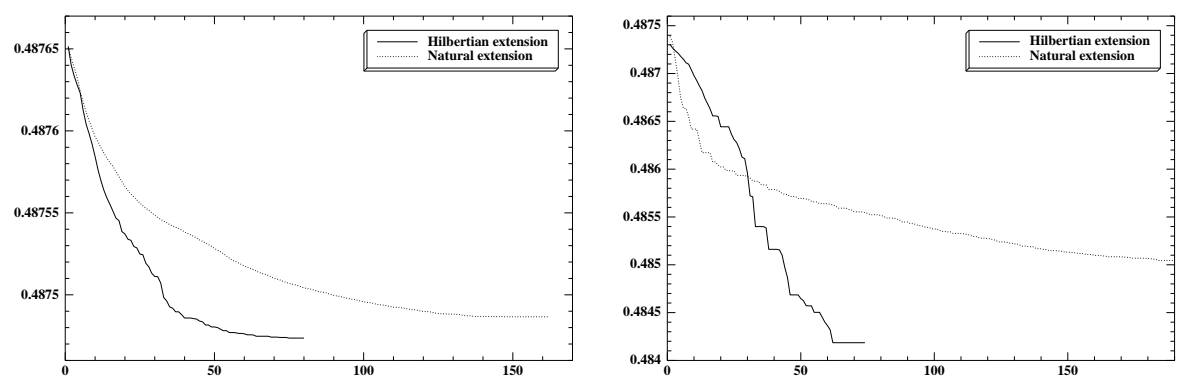

Figure 13: Negative Poisson modulus's evolution of the objective function at the first step (left) with a ratio for the weak material equal to 0.01 and the second step with a ratio of $10^{-5}$ (right).
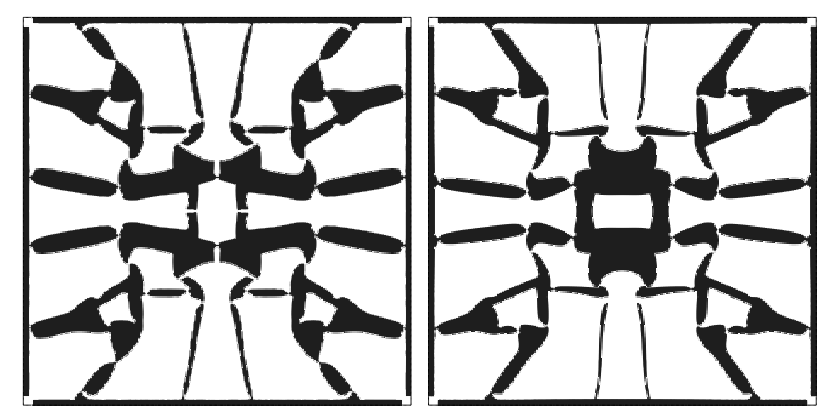

Figure 14: Negative Poisson modulus's optimal shape for the Hilbertian method (left) and the natural method (right) at the end of optimization.

box of Figure 15 for the force inverter and at the top and bottom of Figure 12 for the negative Poisson modulus mechanism). At the location of the input forces (left black box for the force inverter or the left and right walls for the negative Poisson modulus mechanism) the displacement is enforced to be close 

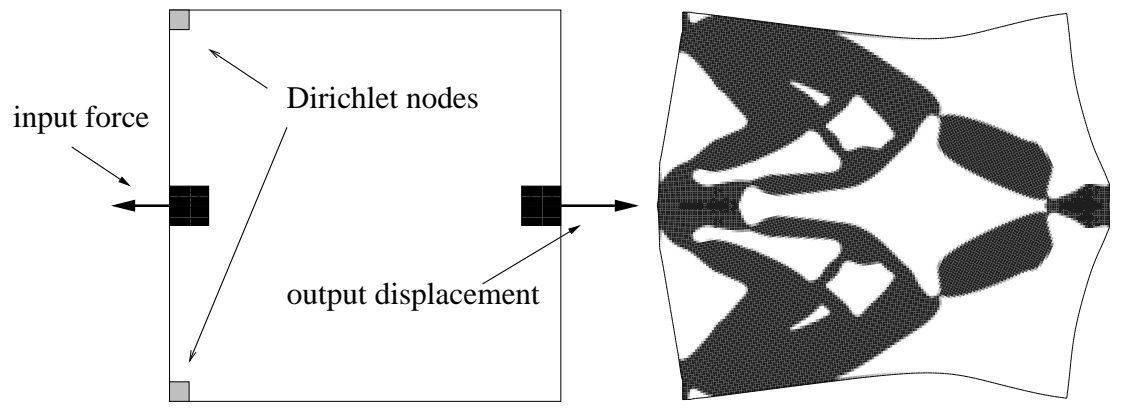

Figure 15: The Force inverter problem and its deformed solution.
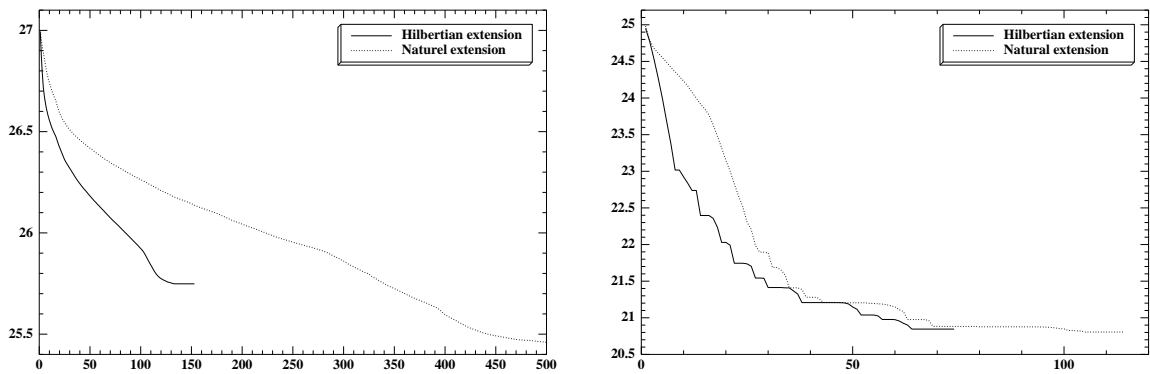

Figure 16: Force inverter's evolution of the objective function for the first step (left) with a ratio for the weak material equal to 0.01 and the second step with a ratio of $10^{-5}$ (right).
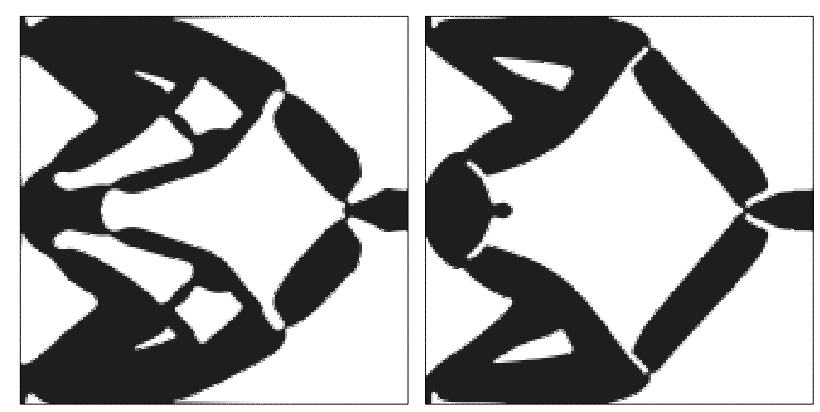

Figure 17: Force inverter's optimal shape for the Hilbertian method (left) and the natural method (right) at the end of optimization.

to zero in order to ensure connectivity of the shapes. Optimization is made in two steps like the 2-d grip of Section 4.5 and the behaviour of the Hilbertian extension with respect to the natural extension is comparable. 


\subsection{Numerical example : A 3-d gripping mechanism}

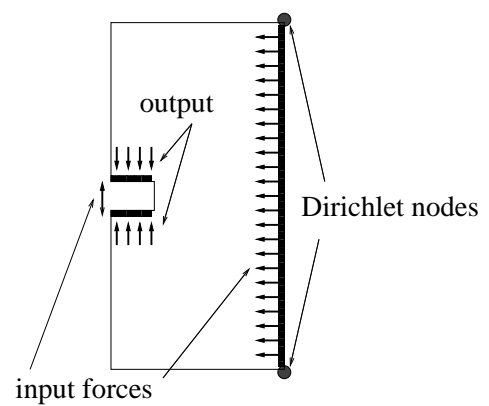

Figure 18: The problem of the 3-d gripping mechanism.
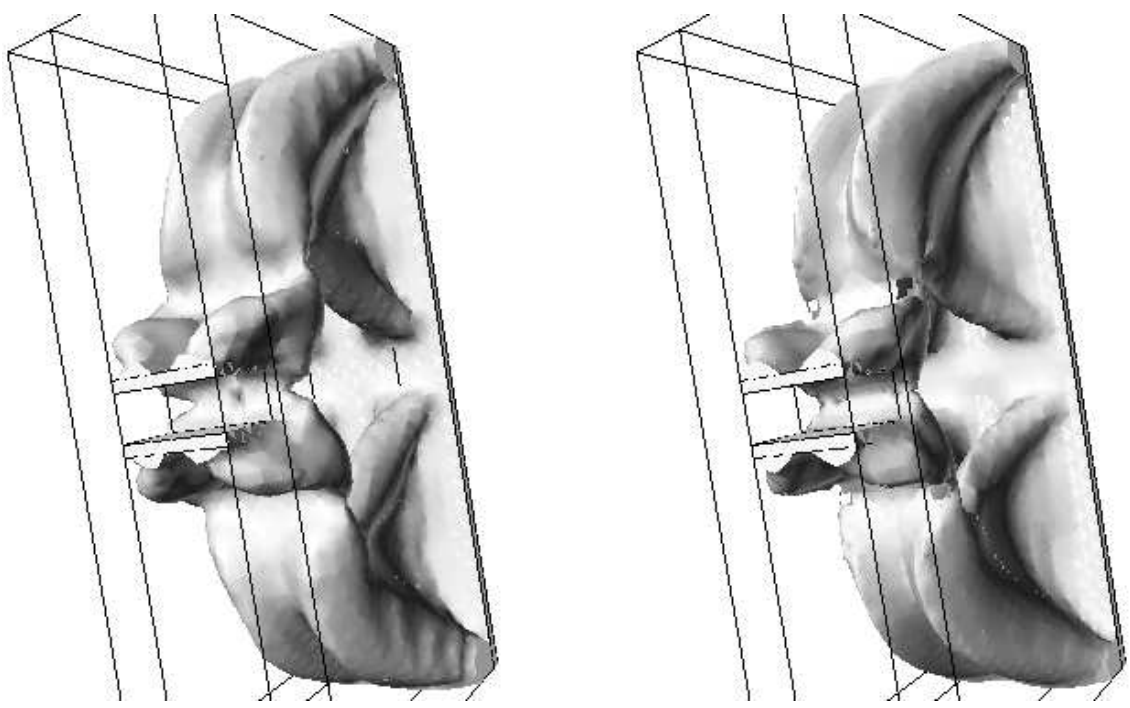

Figure 19: Optimal shape for the Hilbertian method (left) and the natural method (right) (isovalue 0 of the level-set is shown).

The objective function is defined by (8) in Section 4.5. The working domain is a $3 \times 2 \times 6$ rectangle. A uniform pressure load is applied on the plane $x=3$ and the prescribed displacement is localized on a box at the opposite side (see Figure 18 where a cross-section at $y=0$ is shown ). A uniform pressure load (of order 60 percent of the one on the plane $x=3$ ) is also imposed between the jaws of the mechanism so that this mechanism is designed to hold and grip. The Poisson ratio is 0.3 and the Young modulus is 1 . The Lagrange multiplier is set to 3 and the mesh used is $31 \times 21 \times 61$. The ratio of the weak material is set to $10^{-5}$. There is no need here to perform the two-step optimization of the Section 

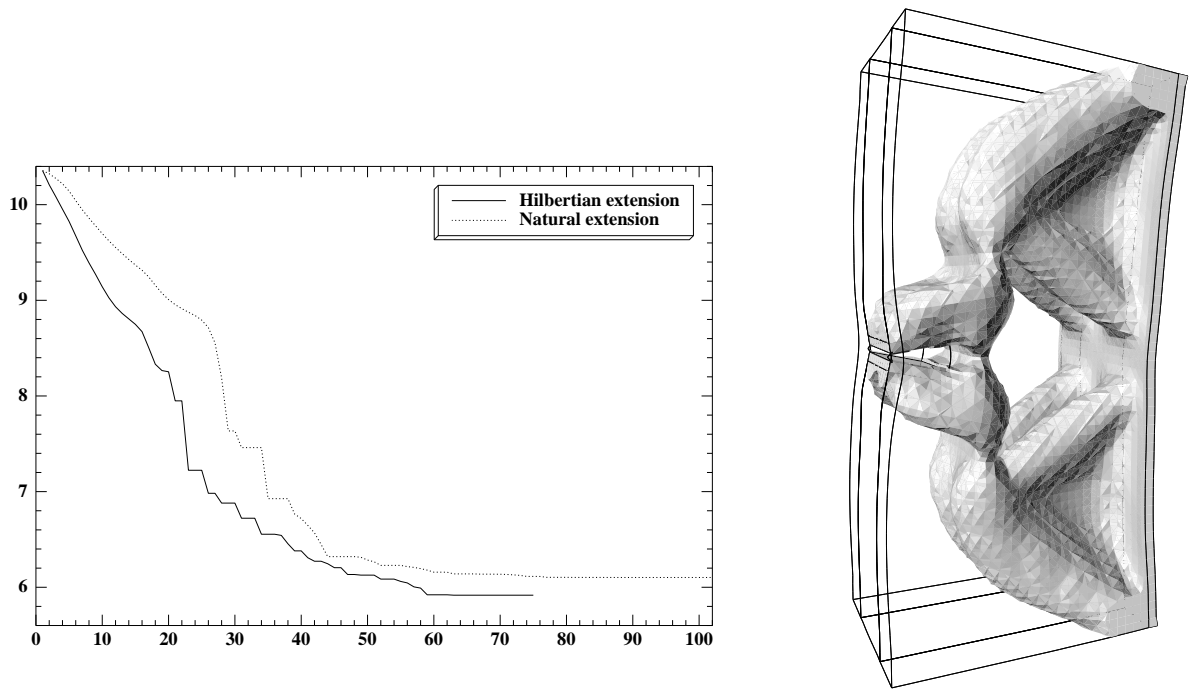

Figure 20: Objective function (left) and displacement of the Hilbertian shape (right) (density of material $\geq 0.3$ is shown).

4.5. The reason why things seems to be simpler in $3-\mathrm{d}$ is that the changes of topology and the hinges are not of the same nature than in 2-d consequently it is believed that throughout the process of optimization, the objective function is more regular in $3-\mathrm{d}$.

\section{Optimizing multiple eigenvalues}

The development above for optimizing the first eigenvalue stands only when this eigenvalue is of multiplicity equal to 1 . When this is not the case, there is no more differentiability of the first eigenvalue with respect to $\theta$ and the above method cannot be applied. Nevertheless it has been proven that $\mathcal{L}$ is directionally differentiable. The velocity extension which endows the space with an Hilbertian structure allows to find a direction of descent. The used algorithm is now a sub-gradient type algorithm. The goal of this Section is two fold :

- Calculate $\mathcal{L}^{\prime}(\theta): W^{1, \infty}(D ; D) \rightarrow \mathbb{R}$ the non-linear collection of directional derivative of $\mathcal{L}$. Show that according to Hadamard's structure theorem $\mathcal{L}^{\prime}(\theta)$ depends only of the value of $\theta \cdot n$ on $\partial \Omega$. Let us denote $j(\theta \cdot n)=\mathcal{L}^{\prime}(\theta)$

- Compute $V^{*}$ such that $j\left(V^{*}\right)=\min _{\|V\|=1} j(V)$ and advance the domain according to $V^{*}$.

It is in the computation of $V^{*}$ that the Hilbertian structure is compulsory needed. 


\subsection{A general theorem about eigenvalue differentiation}

Differentiating eigenvalues when they are multiple is nowadays quite standard. Two different approaches exist, the one of [Kat76] followed by [RC90] and [Lit00]; or the one of [Cox95] (see also [Gou05]) using the subgradient theory of Clarke [Cla90]. All these references give a proof to Theorem 5.2.

Definition 5.1 Define $\Omega_{0}$ as the actual working domain.

$\diamond$ Let $\alpha>0, \beta_{1}>0$ be constants and $\mathbb{L}$ be the space of linear unbounded auto-adjoint operators from $L^{2}\left(\Omega_{0}\right) \rightarrow L^{2}\left(\Omega_{0}\right)$ such that:

$$
\forall L \in \mathbb{L} \quad, \forall u \in H_{D}^{1}\left(\Omega_{0}\right) \quad \beta_{1}\|u\|_{H_{D}^{1}\left(\Omega_{0}\right)}^{2} \geq(L u, u)_{L^{2}} \geq \alpha\|u\|_{H_{D}^{1}\left(\Omega_{0}\right)}^{2}
$$

i.e.: $\mathbb{L}$ is made of operator 'uniformly' coercive with constant $\alpha$ and uniformly continuous with constant $\beta_{1}$

$\diamond$ Let $\beta_{2}>0$ be a constant and $\mathrm{M}$ be the space of continuous linear autoadjoint operators from $L^{2}\left(\Omega_{0}\right) \rightarrow L^{2}\left(\Omega_{0}\right)$ uniformly continuous with constant $\beta_{2}$, ie:

$$
\forall M \in \mathbb{M} \quad, \forall u \in L^{2}\left(\Omega_{0}\right) \quad \beta_{2}\|u\|_{L^{2}\left(\Omega_{0}\right)}^{2} \geq(M u, u)_{L^{2}}
$$

$\diamond$ Define the norm in those two spaces as follows : If $N \in \mathbb{L}$ or $N \in \mathbb{M}$ then

$$
\|N\|=\max _{u \in H_{D}^{1}} \frac{(N u, u)_{L^{2}}}{\|u\|_{H_{D}^{1}}^{2}}
$$

i.e., we endow $\mathbb{M}$ with the natural norm of $\mathbb{L}$

$$
\begin{array}{clc}
\diamond \text { Let } U \subset W^{1, \infty}(D ; D) & \rightarrow \quad \mathbb{L} \times \mathbb{M} \\
\theta & \mapsto(L(\theta), M(\theta))
\end{array}
$$

ping with respect to the norm just defined. Define $L^{\prime}\left(\theta_{0}\right)\left(\right.$ resp $\left.M^{\prime}\left(\theta_{0}\right)\right)$ the differential of $L(\theta)$ (resp $M(\theta)$ ) with respect to $\theta$ at the point $\theta=0$ applied to $\theta_{0}$.

Theorem 5.2 Define $\gamma_{1}(\theta)$ the smallest eigenvalue of the generalized eigenproblem $L(\theta) u=\gamma M(\theta) u$ and $E_{\theta}$ its eigenspace, $E_{\theta}=\operatorname{Ker}\left(L(\theta)-\gamma_{1} M(\theta)\right)$.

Then, for all $\theta_{0} \in W^{1, \infty}(D ; D), \gamma_{1}(\theta)$ is directionally differentiable at the point $\theta=0$ in the direction $\theta_{0}$ and the value of the directional derivative is

$$
\begin{aligned}
\gamma_{1}^{\prime}\left(\theta_{0}\right)= & \min _{\substack{u \in E_{0}\\
}}(M(0) u, u)=1
\end{aligned}
$$

\subsection{Calculus of the directional derivative of $\mathcal{L}$}

We want to apply the general Theorem 5.2 in the shape sensitivity setting. Recall definition of $\tilde{\mathcal{L}}(\theta)$, the functionnal we derivate: 


\section{Definition 5.3}

$\diamond$ Let $T=I d+\theta$ and $\Omega_{\theta}=T \circ \Omega_{0}$

$\diamond$ Let $\tilde{M}(\theta)$ and $\tilde{L}(\theta)$ be defined as : for all $u, v$ in $H_{D}^{1}\left(\Omega_{\theta}\right)$

$$
\begin{aligned}
(\tilde{M}(\theta) u, v) & =\int_{\Omega_{\theta}} \rho u \cdot v \\
(\tilde{L}(\theta) u, v) & =\int_{\Omega_{\theta}} A e(u): e(v)
\end{aligned}
$$

$\diamond$ Let $\tilde{\gamma}_{1}(\theta)$ be the smallest eigenvalue associated to the problem

$$
\tilde{L}(\theta) u=\tilde{\gamma}_{1}(\theta) \tilde{M}(\theta) u
$$

$\diamond$ Let $\tilde{\mathcal{L}}(\theta)=-\tilde{\gamma}_{1}(\theta)+\eta\left|\Omega_{\theta}\right|$

The $\tilde{\mathcal{L}}(\theta)$ (respectively $\tilde{\gamma}_{1}(\theta)$ ) just defined corresponds to what has been denoted $\mathcal{L}\left(\Omega_{\theta}\right)$ (respectively $\gamma_{1}\left(\Omega_{\theta}\right)$ ) in Section 2.1.

Theorem 5.2 cannot be applied to $\tilde{\gamma}_{1}(\theta)$ because the the spaces where the operators $\tilde{L}(\theta)$ and $\tilde{M}(\theta)$ are defined changes with $\theta$. That is why we consider :

\section{Definition 5.4}

$\diamond$ Let $M(\theta) \in \mathbb{M}$ and $L(\theta) \in \mathbb{L}$ be defined as

$$
\begin{aligned}
(M(\theta) u, v) & =\left(\tilde{M}(\theta) u \circ T^{-1}, v \circ T^{-1}\right) \\
(L(\theta) u, v) & =\left(\tilde{L}(\theta) u \circ T^{-1}, v \circ T^{-1}\right)
\end{aligned}
$$

$\diamond$ Let $\bar{\gamma}(\theta)$ be the smallest eigenvalue associated to the problem

$$
L(\theta) u=\bar{\gamma}(\theta) M(\theta) u
$$

We work with those operators instead of the classical one. They are defined on a domain independent of $\theta$ so that the first eigenvalue can be derivated in the sense of theorem 5.2. First it must be proven that the introduced eigenvalue $\bar{\gamma}$ is the same as $\gamma_{1}$ the first eigenvalue of the elasticity problem.

\section{Lemma 5.5}

$\diamond L(\theta)$ and $M(\theta)$ indeed belongs to $\mathbb{L}$ and $\mathbb{M}$.

$\diamond \bar{\gamma}(\theta)$ and $\tilde{\gamma}_{1}(\theta)$ coincides, where $\tilde{\gamma}_{1}$ is defined in definition 5.3 .

\section{Proof of lemma 5.5.}

$\diamond$ Let us first prove that $\tilde{\gamma}_{1}(\theta)=\bar{\gamma}(\theta)$. Using $u \in H^{1}\left(\Omega_{\theta}\right) \Longleftrightarrow v=u \circ T^{-1} \in$ $H^{1}\left(\Omega_{0}\right)$ we have :

$$
\begin{aligned}
\tilde{\gamma}_{1}(\theta)^{-1} & =\max _{u \in H^{1}\left(\Omega_{\theta}\right)} \frac{(\tilde{M}(\theta) u, u)}{(\tilde{L}(\theta) u, u)}=\max _{u \in H^{1}\left(\Omega_{\theta}\right)} \frac{(M(\theta) u \circ T, u \circ T)}{(L(\theta) u \circ T, u \circ T)} \\
& =\max _{v \in H^{1}\left(\Omega_{0}\right)} \frac{(M(\theta) v, v)}{(L(\theta) v, v)}=\bar{\gamma}(\theta)^{-1}
\end{aligned}
$$


$\diamond$ Let us now prove that $L(\theta)$ belongs to $\mathbb{L}$. The fact that $L(\theta)$ is coercive and bounded with respect to the $H_{D}^{1}\left(\Omega_{0}\right)$ norm comes from the fact that $\tilde{L}(\theta)$ is coercive and bounded in the $H_{D}^{1}\left(\Omega_{\theta}\right)$ norm. We have to show that these constant of coercivity and boundedness are uniform in $\theta$. Let's introduce the tensor $A$ which has the symmetries of the elasticity :

$$
A^{i j k l}=A^{j i k l}=A^{i j l k}=A^{k l i j}
$$

such that $A^{i j k l}\left(\partial_{j} u^{i}\right)\left(\partial_{l} v^{k}\right)=A e(u): e(v)$. We use the standard tool of shape sensitivity, namely a change of variable :

$$
\begin{aligned}
(L(\theta) u, v) & =\int_{\Omega_{\theta}} A^{i j k l} \partial_{j}\left(u \circ T^{-1}\right)^{i} \partial_{l}\left(v \circ T^{-1}\right)^{k} \\
& =\int_{\Omega_{0}}|\operatorname{det} \nabla T| A^{i j k l}\left(\partial_{s} u^{i}\right)\left(\partial_{j}\left(T^{-1}\right)^{s}\right)\left(\partial_{m} v^{k}\right)\left(\partial_{l}\left(T^{-1}\right)^{m}\right) \\
& =\int_{\Omega_{0}} l(\theta)^{i s k m}\left(\partial_{s} u^{i}\right)\left(\partial_{m} v^{k}\right)
\end{aligned}
$$

With

$$
l(\theta)^{i s k m}=A^{i s k m}+\left(\partial_{l} \theta^{l}\right) A^{i s k m}-A^{i j k m}\left(\partial_{j} \theta^{s}\right)-A^{i s k l}\left(\partial_{l} \theta^{m}\right)+o\left(\|\theta\|_{W^{1, \infty}}\right)
$$

Given $\alpha$ and $\beta$ the coercivity and continuity constant of $L(0)$, there exist $\eta>0$ such that for every $\theta$ with $\|\theta\|_{W^{1, \infty}}<\eta$, we have :

$$
\forall u \in H_{D}^{1}\left(\Omega_{0}\right) \quad 2 \beta_{0}\|u\|_{H_{D}^{1}\left(\Omega_{0}\right)}^{2} \geq(L(\theta) u, u)_{L^{2}} \geq \frac{\alpha_{0}}{2}\|u\|_{H_{D}^{1}\left(\Omega_{0}\right)}^{2}
$$

We then verify the hypothesis of definition 5.1 with $\alpha_{1}=\alpha / 2, \beta_{1}=2 \beta$ and $U=\left\{\theta\right.$ s.t. $\left.\|\theta\|_{W^{1, \infty}}<\eta\right\}$. An analog development stands for $M$.

We can now apply theorem 5.2 to $\bar{\gamma}(\theta)=\tilde{\gamma}_{1}(\theta)$ and end with the following result.

Theorem 5.6 Recalling definition 5.3, $\tilde{\mathcal{L}}(\theta)=-\tilde{\gamma}_{1}(\theta)+\eta\left|\Omega_{\theta}\right|$ is directionally differentiable with respect to $\theta$ and its directionally derivative at $\theta=0$ in the direction $\theta_{0}$ is given by

$$
\begin{aligned}
\mathcal{L}^{\prime}\left(\theta_{0}\right)= & \max _{\substack{u \in E_{0} \\
\int_{\Omega_{0}} \rho u \cdot u=1}} \int_{\partial \Omega_{0}}\left(\theta_{0} \cdot n\right)(-v(u, u)+\eta) \\
&
\end{aligned}
$$

where $v(\cdot, \cdot)$ is a bilinear functional defined by

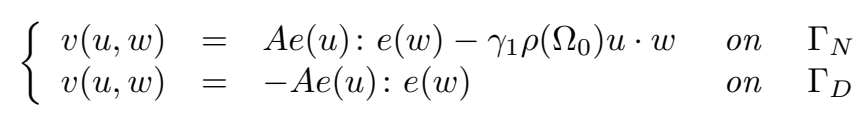

and where $E_{0}$ is the first eigenspace associated to $\gamma_{1}\left(\Omega_{0}\right)=\tilde{\gamma}(0)$ the smallest eigenvalue for $\theta=0$. 
One can of course verify that the formula when the dimension of the eigenspace is greater than one is the same that the formula when the dimension is equal to one.

\section{Proof of theorem 5.6}

We apply Theorem 5.2 to $\tilde{\gamma}_{1}(\theta)=\bar{\gamma}(\theta)$ in order to calculate $\mathcal{L}^{\prime}\left(\theta_{0}\right)$. The calculus above for $L$ (and the same for $M$ ) shows that

$$
\begin{aligned}
\left(L^{\prime}(\theta) u, v\right) & =\int_{\Omega_{0}}\left(\left(\partial_{l} \theta^{l}\right) A^{i s k m}-A^{i j k m}\left(\partial_{j} \theta^{s}\right)-A^{i s k l}\left(\partial_{l} \theta^{m}\right)\right)\left(\partial_{s} u^{i}\right)\left(\partial_{m} v^{k}\right) \\
& =\int_{\Omega_{0}} A^{i s k m}\left(\partial_{l} \theta^{l}\right)\left(\partial_{s} u^{i}\right)\left(\partial_{m} v^{k}\right)-A^{i s k m}\left(\partial_{s} \theta^{l}\right)\left(\partial_{l} u^{i}\right)\left(\partial_{m} v^{k}\right) \\
\left(M^{\prime}(\theta) u, v\right) & =\int_{\Omega_{0}}\left(\partial_{l} \theta^{l}\right) \rho u^{i} v^{i}
\end{aligned}
$$

Applying Theorem 5.2 gives

$$
\begin{aligned}
\mathcal{L}^{\prime}=\max _{\substack{u \in E_{0} \\
\int_{\Omega_{0}} \rho u \cdot u=1}} & -\int_{\Omega_{0}} A^{i s k m}\left(\partial_{l} \theta^{l}\right)\left(\partial_{s} u^{i}\right)\left(\partial_{m} v^{k}\right)+A^{i s k m}\left(\partial_{s} \theta^{l}\right)\left(\partial_{l} u^{i}\right)\left(\partial_{m} v^{k}\right) \\
& +\int_{\Omega_{0}} A^{i s k m}\left(\partial_{m} \theta^{l}\right)\left(\partial_{s} u^{i}\right)\left(\partial_{l} v^{k}\right)+\left(\partial_{l} \theta^{l}\right) \rho u^{i} v^{i}+\eta\left(\partial_{l} \theta^{l}\right)
\end{aligned}
$$

Performing an integration by part on $\theta$, the term in $\int_{\Omega_{0}}$ is equal to :

$$
\theta^{l} A^{i s k m}\left[\partial_{l}\left(\partial_{s} u^{i} \partial_{m} u^{k}\right)-\partial_{s}\left(\partial_{l} u^{i} \partial_{m} u^{k}\right)-\partial_{m}\left(\partial_{s} u^{i} \partial_{l} u^{k}\right)\right]-\theta^{l} \gamma_{1} \rho \partial_{l}\left(u^{i} u^{i}\right)
$$

Some algebra used in coordination with $\gamma_{1} \rho u=-\operatorname{div} A e(u)$ allows us to conclude that this term is equal to zero. The remaining term is then equal to:

$$
\begin{aligned}
\mathcal{L}^{\prime}(\theta)=\max & \quad \int_{\partial \Omega_{0}}(\theta \cdot n)\left[-A^{i s k m}\left(\partial_{s} u^{i}\right)\left(\partial_{m} u^{k}\right)+\gamma_{1} \rho u \cdot u+\eta\right] \\
+ & \int_{\partial \Omega_{0}} A^{i s k m} \theta^{l} n^{m}\left(\partial_{s} u^{i}\right)\left(\partial_{l} u^{k}\right)+A^{i s k m} \theta^{l} n^{s}\left(\partial_{l} u^{i}\right)\left(\partial_{m} u^{k}\right)(12
\end{aligned}
$$

On the Neumann part of the boundary we use $A e(u) \cdot n=0$ and the definition of $C$ to conclude that:

$$
A^{i s k m}\left(\partial_{s} u^{i}\right) n^{m}=0=A^{i s k m}\left(\partial_{m} u^{k}\right) n^{s} .
$$

On the Dirichlet part of the boundary we use $u=0$ so that $\nabla u=\frac{\partial u}{\partial n} \otimes n$ and

$$
(\theta \cdot n)\left(\partial_{m} u^{k}\right)=\theta^{l} n^{m}\left(\partial_{l} u^{k}\right) \text { and }(\theta \cdot n)\left(\partial_{s} u^{i}\right)=\theta^{l} n^{s}\left(\partial_{l} u^{i}\right) .
$$

So that

$$
\mathcal{L}^{\prime}(\theta)=\max \int_{\partial \Omega_{0}}(\theta \cdot n)\left[-A e(u): e(u)+\gamma_{1} u \cdot u+\eta\right]+2 \int_{\Gamma_{D}}(\theta \cdot n) A e(u): e(u)
$$


Remark 5.7 Assumed regularity of $\Omega_{0}$ We need regularity on the domain $\Omega_{0}$ in two occurrences. The first one is when we perform a change of variable ((9) in the demonstration of lemma 5.5), and the second one is when we perform an integration by part ((11) in the demonstration of Theorem 5.6).

The change of variable is compulsory in order to prove the derivability. It is sufficient to suppose $\Omega_{0}$ Lipschitz in order to be able to perform it.

The integration by part is only used in order to express the directional derivative (10) as an integral over $\partial \Omega_{0}$ (see (12)). Performing this integration by part is asking a lot of regularity on $\Omega_{0}$, because the eigenvector $u$ must be regular enough ie, $u \in H^{1}\left(\partial \Omega_{0}\right)$. But this integration by part is not needed if the only goal is to prove the directional derivability.

\subsection{Calculating $V^{*}$}

The goal of this subsection is to calculate $V^{*}$ the minimizer to

$$
\min _{\|V\|=1} \max _{\substack{u \in E_{0} \\ \int_{\Omega_{0}} u \cdot u=1}} \int_{\partial \Omega_{0}} V(-v(u, u)+\eta)
$$

Where $v(\cdot, \cdot)$ is defined in Theorem 5.6. We will prove in this Section that this is an semidefinite programming problem (SDP) in low dimension that is easily solved. The use of SDP programming for eigenvalue optimization is classical, the goal of this Section is to show that solving this SDP problem is a very easy task thanks to the Hilbertian structure endowed by the velocity regularization. A good introduction to SDP programming is [VB96] and the references therein. Only some basic facts about SDP problems have been recalled here

\section{Definition 5.8}

$\diamond$ Let $Y$ be an unknown vector, Give $Y_{0}$ a vector and $E(Y)$ a matrix whose coefficients depend linearly on $Y$. Let $\geq 0$ stand for 'symmetric positive'. An SDP problem is of the form:

$$
\min _{E(Y) \geq 0} Y^{T} Y_{0}
$$

$\diamond$ SDP problems are efficiently solvable by duality methods. In order to insure that there is no gap of duality, a sufficient condition is to find $Y_{1}$ a strictly primal feasible point, i.e.: such that $E\left(Y_{1}\right)>0$ (definite positive).

We now need to introduce the semidefinite programming problem we will work on.

Definition 5.9 Recall definition 4.1 of the scalar product $(\cdot, \cdot)_{H^{1}}$

$\diamond$ Define $\left(e_{i}\right)_{i=1 . . d}$ an orthonormal basis of $E_{0}$ for the scalar product

$$
(u, w)=\int_{\Omega_{0}} \rho u \cdot v
$$


$\diamond$ Define $\left(a_{i j}\right)_{i, j=1 . . d}$ and $c$ as

$$
\left(a_{i j}, X\right)_{H^{1}}=\int_{\partial \Omega_{0}}-X v\left(e_{i}, e_{j}\right) \quad \text { and } \quad(c, X)_{H^{1}}=\int_{\partial \Omega_{0}} X \eta
$$

$\diamond$ Define $h_{k}$ an orthonormalized basis of $\operatorname{Span}\left(a_{i j}, c\right)_{i, j}$ for the scalar product $(,)_{H^{1}}$. Let $m$ be the dimension of this space and let $\left(a_{i j}^{k}\right)_{k=1 . . m}\left(\operatorname{resp}\left(c^{k}\right)_{k=1 . . m}\right)$ be the coordinates of $a_{i j}($ resp $c)$ on the basis $\left(h_{k}\right)_{k=1 . . m}$.

$\diamond$ For any $X=\left(X_{1}, . ., X_{m}\right)$ and $(z, w) \in \mathbb{R}^{2}$, let $Y=[X, w, z]$.

$\diamond$ Let $A(X)$ be the $d \times d$ matrix $A(X)_{i j}=a_{i j}^{k} X_{k}$ let $C(X)=c^{k} X_{k}$ and let

$$
D(Y)=\left[\begin{array}{cc}
-A(X)+z I d & 0 \\
0 & -C(X)-z+w
\end{array}\right] \quad E(Y)=\left[\begin{array}{ccc}
D(Y) & 0 & 0 \\
0 & I d & X \\
0 & X^{T} & 1
\end{array}\right]
$$

The coefficients of $E$ depends linearly on $Y=[X, w, z]$

$\diamond$ Let $Y^{*}=\left[X^{*}, w^{*}, z^{*}\right]$ be the solution of the following SDP problem :

$$
\min _{E(Y) \geq 0} w
$$

Theorem 5.10

$\diamond V^{*}$, the minimizer of the problem (13) is given by

$$
V^{*}=\sum_{k=1}^{m} V_{k} h_{k}
$$

where the vector $X^{*}=\left[V_{1}, . ., V_{k}\right]$ is defined as a solution of the Semi-Definite problem (14).

$\diamond$ The problem $\min _{E(Y) \geq 0} w$ is strictly feasible, SDP programming can be applied. $\diamond V^{*}$ (or equivalently $\left.X^{*}\right)$ is attained.

\section{Proof}

$\diamond$ We transform the problem (13) into (14) by using the fact that $v(\cdot, \cdot)$ is bilinear (see Theorem 5.6 for the definition of $v$ )

$$
\begin{aligned}
& \min _{\|V\|_{H^{1}}=1} \max _{\substack{u \in E_{0} \\
\int_{\Omega_{0}} \rho u \cdot u=1}}\left(\int_{\partial \Omega_{0}} V[-v(u, u)+\eta]\right) \\
= & \min _{\|V\|_{H^{1}}=1} \max _{\sum_{i=1}^{d} \lambda_{i}^{2}=1}\left(\int_{\partial \Omega_{0}} V\left[-\lambda_{i} \lambda_{j} v\left(e_{i}, e_{j}\right)+\eta\right]\right) \\
= & \min _{\|V\|_{H^{1}}=1} \max _{\sum_{i=1}^{d} \lambda_{i}^{2}=1}\left[\lambda_{i} \lambda_{j}\left(V, a_{i j}\right)_{H^{1}}+(V, c)_{H^{1}}\right] \\
= & \min _{\|V\|_{H^{1}}=1} \max _{\sum_{i=1}^{d} \lambda_{i}^{2}=1}\left[\left(V, h_{k}\right)_{H^{1}}\left(\lambda_{i} \lambda_{j} a_{i j}^{k}+c^{k}\right)\right]
\end{aligned}
$$


So that $V^{*}$ is a minimizer of (13) if and only if $X_{k}^{*}=\left(V^{*}, h_{k}\right)_{H^{1}}$ are minimizer of the following problem

$$
\min _{\sum X_{k}^{2} \leq 1} \max _{\sum_{i=1}^{d} \lambda_{i}^{2}=1}\left[\lambda_{i} \lambda_{j} a_{i j}^{k} X_{k}+c^{k} X_{k}\right]
$$

$\diamond$ Showing that (15) is equivalent to (14) is a standard issue of SDP programming: The condition $E([X, w, z]) \geq 0$ is equivalent to $(X, X) \leq 1$ and $D([X, w, z]) \geq 0$. The condition $D([X, w, z]) \geq 0$ is equivalent to $z I d \geq A(X)$ and $w \geq z+C(X)$. So that $E([X, w, z]) \geq 0$ is equivalent to:

$$
\left(\sum_{k} X_{k}^{2} \leq 1\right) \text { and }\left(w \geq z+X_{k} c^{k}\right) \text { and }\left(z \geq \max _{\sum_{i=1}^{d} \lambda_{i}^{2}=1}\left[\lambda_{i} \lambda_{j} a_{i j}^{k} X_{k}\right]\right) \text {. }
$$

So that minimizing $w$ with the above condition is equivalent to finding $X$ in the problem (15).

$\diamond$ Choosing $X=0, z>0$ and $w>z$ gives a $[X, w, z]$ for which $F([X, w, z])>0$ the problem is then strictly feasible, an extended Slater's condition holds and the dual problem (in term of semi-definite duality) have the same extremal value.

$\diamond$ The condition $\sum_{k} X_{k}^{2} \leq 1$ ensures that $X$ is bounded and that every minimizing sequence converges up to a subsequence. The maximum is indeed attained

Remark 5.11 The SDP problem is not difficult to solve, recall that $d$ is the dimension of the first eigenspace, then $[X, w, z]$ is of dimension lower or equal to $\frac{d(d+1)}{2}+3$ and the matrix $E$ is a $\frac{d(d+3)}{2}+3$ square matrix.

\section{$6 \quad$ Numerical results}

\subsection{The 3-d eigenvalue of a beam}

We naturally set our problem in 3-d with symmetries, where we are sure to obtain a multiplicity of the first eigenvalue greater than one. The first example which will be called the 'big domain' problem is a $3 \times 3 \times 1$ rectangle discretized with a $21 \times 21 \times 23$ mesh. A zero displacement boundary condition is imposed on the plane $z=0$ and four cells on the middle of the plane $z=1$ are not subject to optimization and are 50 times heavier (see Figure 21). Since the domain is symmetric, the shapes are expected to keep a first eigenvalue of dimension at least 2 along the iterations. The Young's modulus is set to 1 and Poisson ratio to 0.3 . In the void, the density $\rho$ is set to 0 and the parameter $\varepsilon$ is equal to $10^{-5}$. The second problem is the same than the first except that the rectangle is of dimension $0.6 \times 0.6 \times 1$ (discretized by a $15 \times 15 \times 43$ mesh) with a mass tip that is 200 times heavier. The second problem will be called in this Section the 'narrow domain' problem.

$\diamond$ Discussion about the big domain problem The Lagrange multiplier being set to $5.3 \times 10^{-8}$, Figure 23 is a display of the evolution of the three smallest 

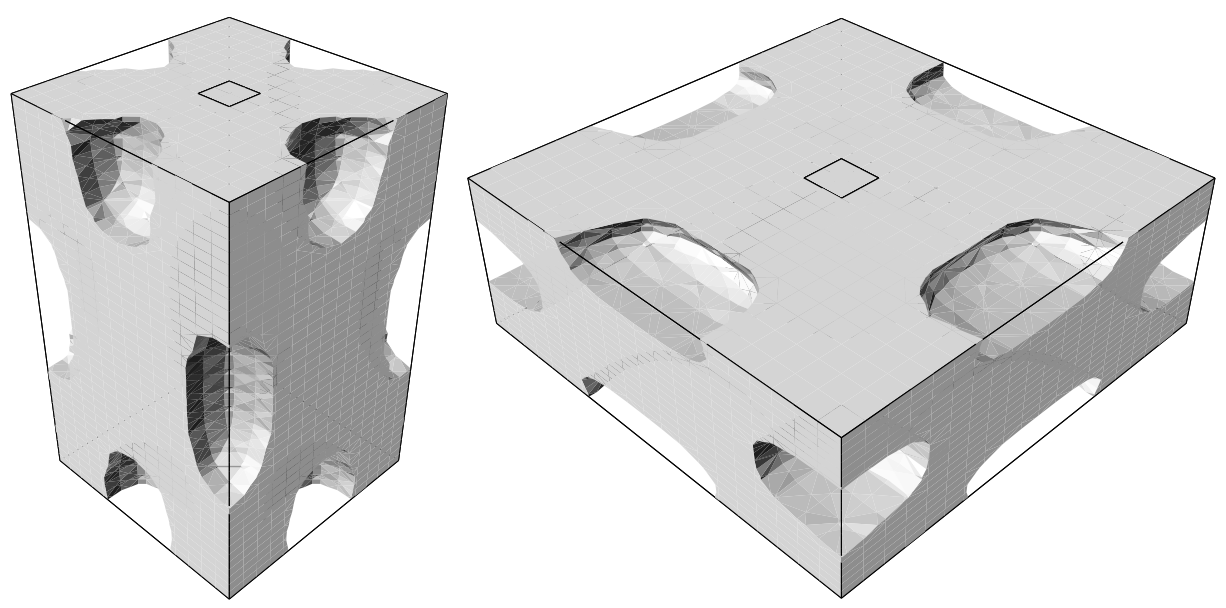

Figure 21: Initialization for narrow domain (left) and for the big domain (right).
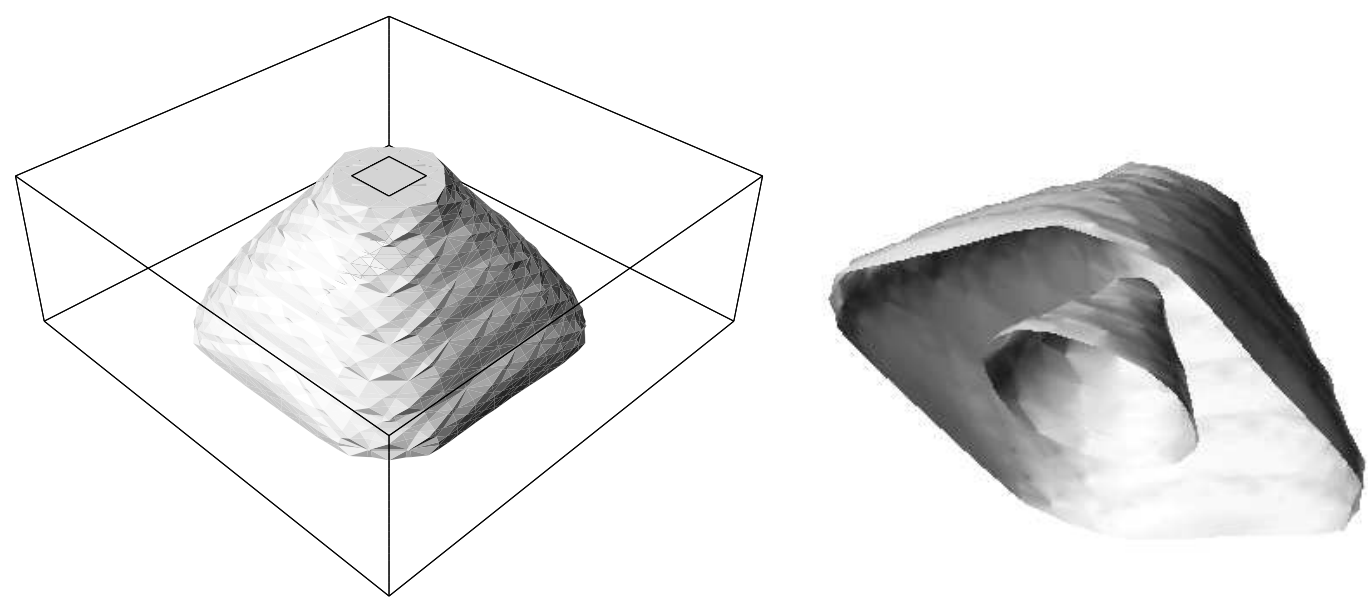

Figure 22: The optimal shape for the first eigenvalue in a big domain (left) and its boundary (right).

eigenvalues. There is one eigenvalue that is always (except on iterations 22 to 28 ) of multiplicity two and one which is of multiplicity one. The eigenvalue of multiplicity one corresponds to an eigenvector which is localized on the heavy cells and that interfere in the optimization process. The Figure on the left of Figure 23 shows the evolution of the eigenvalues. On each iteration, the value of $d$ the dimension of the subspace of the first eigenvalue is shown. The expected behaviour of the algorithm can be verified.

$\diamond$ Discussion about the narrow domain problem As can be seen on Figure 25 (left), the global evolution of the algorithm is as follows: First reinforce 

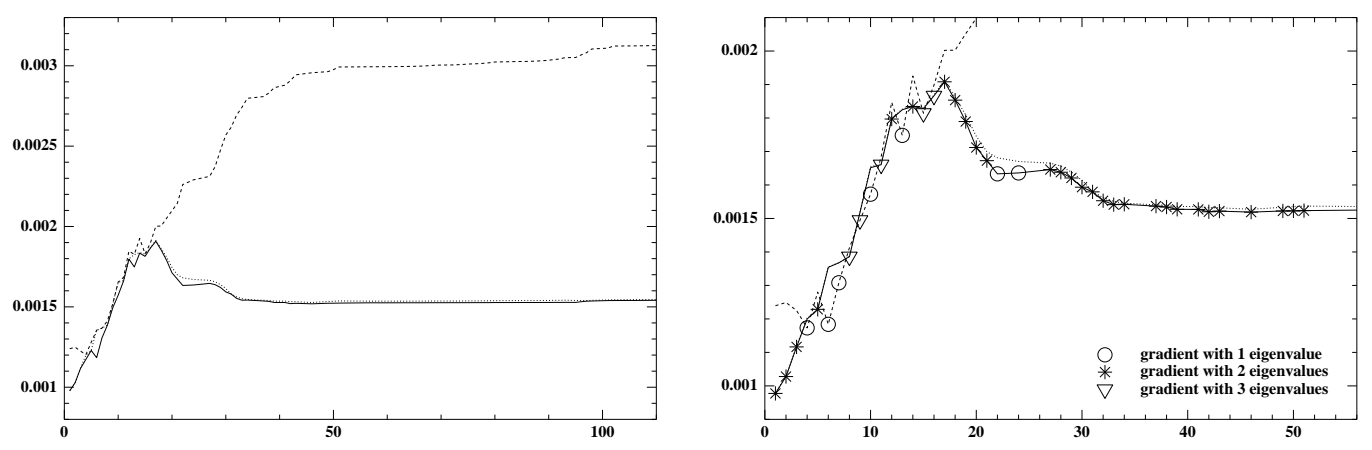

Figure 23: Evolution of the three smallest eigenvalue (left) and an interpretation (right) for the big domain.

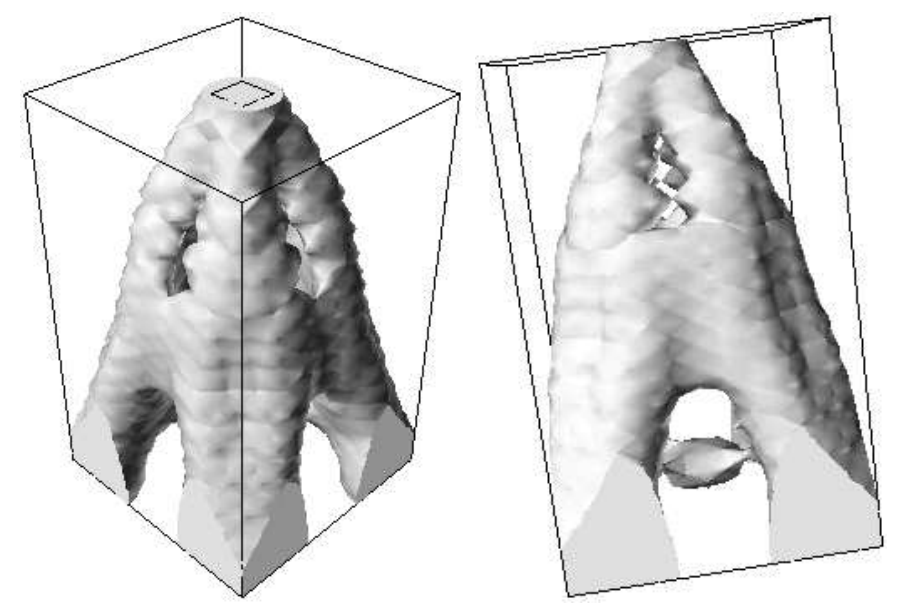

Figure 24: The optimal shape for the first eigenvalue in a narrow domain (isovalue 0.2 of the density is drawn).

the structure so that the first eigenvalue raises and then optimize the weight of the structure. The Lagrange multiplier is set to $10^{-8}$ for this example.

$\diamond$ Discussion about the symmetries None of the two problems give rise to radially-symmetrical shapes. For the narrow-domain problem it can be easily understood by the fact that the shape is constrained into a box. For the bigdomain problem one can advance an explanation based on a mesh-effect. But it is known that there exist symmetric problem whose solutions do not respect the symmetries. We still do not know if the optimal shape is or is not radiallysymmetric for this problem. 

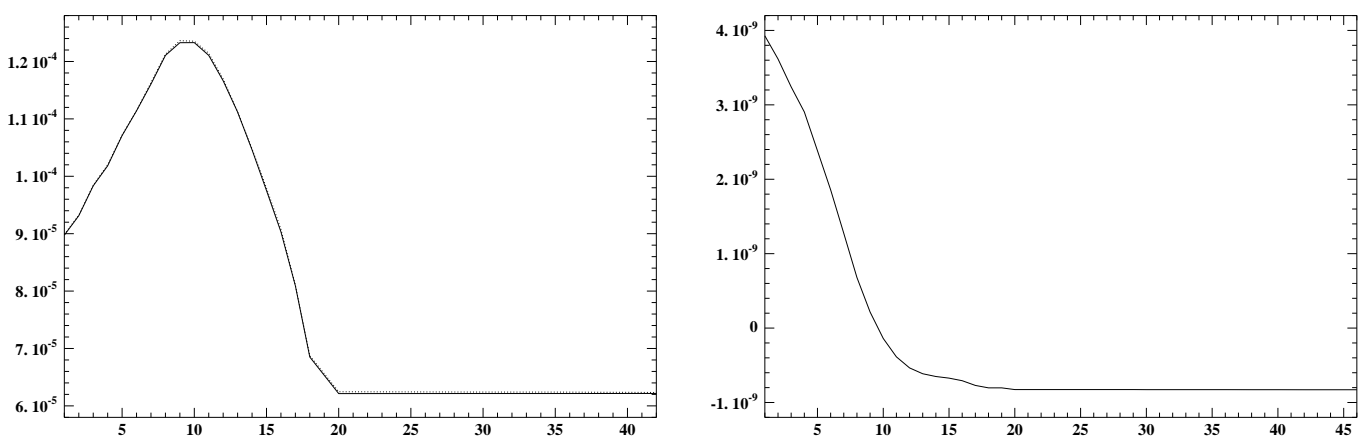

Figure 25: Evolution of the two smallest eigenvalue (left) and the objective function (right) for the narrow domain.

\subsection{The short cantilever}

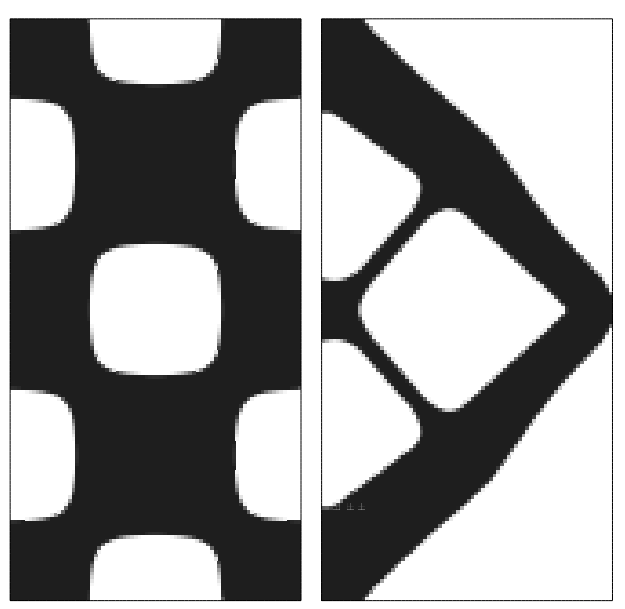

Figure 26: Initialization and optimal shape for the first eigenvalue of a short cantilever.

We run our algorithm on a vibrating cantilever that is the same test case than the one of Section 4.3 except that the working domain is of size $1 \times 2$ discretized with a regular $80 \times 160$ mesh. The other parameters that have changed are the Lagrange multiplier which is set to 0.3 and the heavier mass which has a density 80 times heavier. This test case was introduced in [AJ05] where the authors pointed out the appearance of multiple eigenvalues. It is not the exact same test, because when the test of [AJ05] has been run, the improvement of the multiple eigenvalue method were not as obvious as in the test presented here.

The test is run with the standard single eigenvalue optimization (only one 


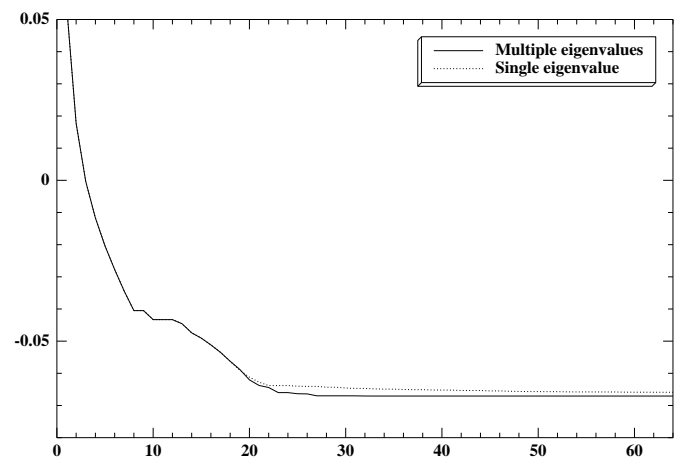

Figure 27: Evolution of the objective function for the two different methods.
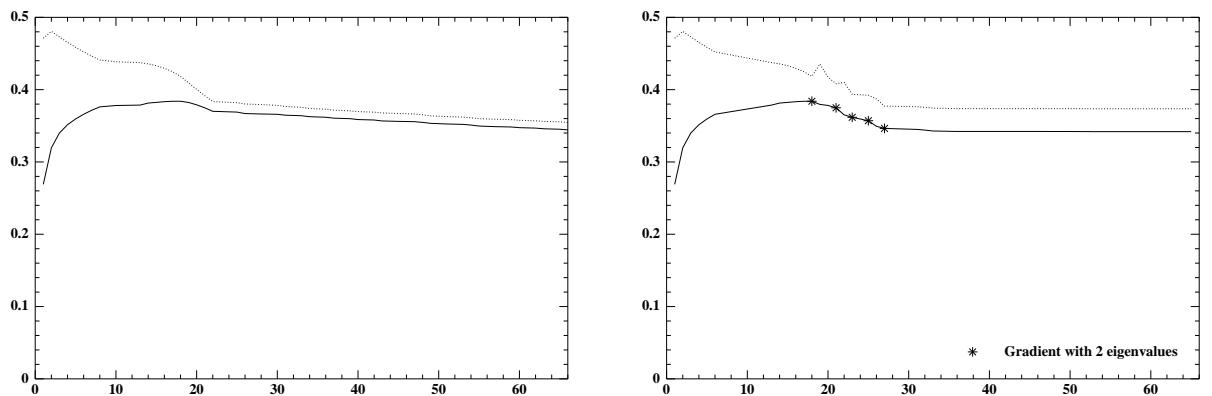

Figure 28: Evolution of the two smallest eigenvalues for the Single eigenvalue optimization (left) and the Multiple eigenvalue optimization (right).

eigenvalue is taken in account during optimization) and the multiple eigenvalue optimization processes. Figure 27 shows the evolution of the objectives functions for the two different processes.

At iteration $n$, the algorithm considers an eigenvalue to be of multiplicity $d$ if and only if the relative differences $\gamma_{d} / \gamma_{1}-1 \leq \epsilon_{0}<\gamma_{d+1} / \gamma_{1}-1$. Where $\varepsilon_{0}$ is a user-defined criterion. If the shape computed at iteration $n+1$ is not better than the shape computed at iteration $n$ and if the eigenvalue at iteration $n$ is multiple then the parameter $\varepsilon_{0}$ is decreased. The parameter $\varepsilon_{0}$ is set to 10 percent at the beginning of the optimization process. This explains the behaviour of the algorithm in Figure 28 (right).

It can be seen in Figure 28 (left) that when the first eigenvalue is considered to be always of multiplicity one, the two first eigenvalues have a tendency to merge and the algorithm cannot improve the shape. If we follow the branches of eigenvalues according to the modes, we would see that the smallest eigenvalue does not correspond to the same modes during optimization. ie, the algorithm optimizes one mode at iteration $n$ and an other one at iteration $n+1$. This 
is standard of optimization with respect to a maximum of a function when the maximum is multiple and it is well-known to slow down the algorithm.

\section{Conclusion}

The velocity-regularization method presented here improves the speed and accuracy of the level set method and extends it to new problems. Thanks to the Hilbertian method, three issues can be dealt with : The extension, the regularization and the endowment of an Hilbertian structure (see discussion in Section $4.2)$.

The extension issue (i.e. extending everywhere a velocity which is only defined on the boundary) is only related to the speed of the algorithm. The 2-d compliant cantilever of Section 4.4 which shows the mesh-independence of the Hilbertian method is a good example of the improvements this new method brings to the level set algorithm.

The regularization issue is about dealing with more regular velocities. It is indeed an improvement as can be seen in the mechanism examples because it allows to improve the accuracy of the optimal shape. It allows to improve too the speed of the algorithm by diffusing the peaks of the velocity in the vicinity of the peak. The only test for which velocity regularization is not as efficient in terms of accuracy as the natural extension is the first step of the optimization procedure of the 2-d mechanism of Section 4.5 (see figure 8 (left)). This can be explained by the fact that the adjoint is not computed with an enough accurate precision when the ratio of the weak material is too high. Sadly for 2-d tests which need the computation of an adjoint, it still seems compulsory to perform the two-steps optimization procedure. It was seen in Section 4.7 that this trick is not needed in 3-d.

Because the velocity-regularization endows the problem with an Hilbertian structure, it allows to apply the level set method of optimization for several problems that are not differentiable but whose directional derivative exists. The computation of the descent direction relies on an SDP problem. The transformation of the steepest descent algorithm into an SDP problem can be made because the directional derivative of the problem is a maximum of quadratic functions over a sphere. This is the case for the eigenvalue problem, for the robust compliance problem and for the buckling load problem which are all based on generalized eigenvalues problem. The investigation of the two latest problems and application of the Hilbertian method to those issues is in progress.

\section{Acknowledgments}

I deeply thank Grégoire Allaire for his advising this work, his useful and always pertinents comments and suggestions; François Jouve for his programming skills and useful critics. Every figures were obtained by François Jouve's personal software. 


\section{References}

[ABFJ97] G. Allaire, E. Bonnetier, G. Francfort, and F. Jouve. Shape optimization by the homogenization method. Nümerische Mathematik, $76: 27-68,1997$.

[AGJT05] G. Allaire, F.de Gournay, F. Jouve, and A-M. Toader. Structural optimization using topological and shape sensitivity via a level set method. Control and Cyb., 2005. to appear.

[AJ05] G. Allaire and F. Jouve. A level-set method for vibrations and multiple loads in structural optimization. Comp. Meth. Appl. Mech. Engrg., 2005.

[AJT04] G. Allaire, F. Jouve, and A-M Toader. Structural optimization using sensitivity analysis and a level-set method. J. comput. Phys., 194(1):363-393, 2004.

[All01] G. Allaire. Shape optimization by the homogenization method. Springer Verlag, New York, 2001.

[Ben95] M. Bendsoe. Methods for optimization of structural topology, shape and material. Springer Verlag, New York, 1995.

[Ber04] P. Bernardoni. Outils et méthode de conception de structure mécaniques à déformations et actionnements répartis. $\mathrm{PhD}$ thesis, Université Paris VI, 2004.

[BHR04] M. Burger, B. Hackl, and W. Ring. Incorporating topological derivatives into level set methods. J. Comput. Phy., 194(1):344-362, 2004.

[BK88] M. Bendsoe and N. Kikuchi. Generating optimal topologies in structural design using a homogeneization method. Comp. Meth. Appl. Mech. Eng., 71:197-227, 1988.

[BS03] M. Bendsoe and O. Sigmund. Topology Optimization. Theory, Methods, and Applications. Springer Verlag, New York, 2003.

[Bur03] M. Burger. A framework for the construction of level set methods for shape optimization and reconstruction. Interfaces and Free Boundaries, 5:301-329, 2003.

[CC03] A. Cherkaev and E. Cherkaeva. Principal compliance and robust optimal design. J. Elasticity, 72:71-98, 2003.

[Che00] A. Cherkaev. Variational Methods for Structural Optimization. Springer Verlag, New York, 2000.

[Cla90] F. H. Clarke. Optimization and Nonsmooth Analysis. SIAM, classic in appl. math. edition, 1990. 
[Cox95] J. Cox. The generalized gradient at a multiple eigenvalue. Journal of Functional Analysis, 1995.

[ES94] H. Eschenauer and A. Schumacher. Bubble method for topology and shape optimization of structures. Structural Optimization, 8:42-51, 1994.

[GGM01] S. Garreau, P. Guillaume, and M. Masmoudi. The topological asymptotic for pde systems: the elasticity case. SIAM J. Control Optim., 39(6):1756-1778, 2001.

[Gou05] F.de Gournay. Optimisation de formes par la méthode des lignes de niveaux. PhD thesis, Ecole Polytechnique, 2005.

[Kat76] T. Kato. Perturbation theory for linear operators. Springer-Verlag, New York, 1976.

[Lit00] G-W. Litvinov. Optimization in elliptic problems with applications to mechanics of deformable bodies and fluid mechanics, volume 119 of Operator Theory. Birkhauser, 2000.

[MP01] B. Mohammadi and O. Pironneau. Applied shape optimization for fluids. Oxford Clarendon Press, 2001.

[NS04] S.A. Nazarov and Y. Sokolovski. The topological derivative of the dirichlet integral under the formation of a thin bridge. Siberian. Math. J., 45(2):341-355, 2004.

[OS88] S. Osher and J-A. Sethian. Front propagating with curvature dependent speed: algorithms based on hamilton-jacobi formulations. $J$. Comput. Phys., 78:12-49, 1988.

[OS01] S. Osher and F. Santosa. level-set methods for optimization problems involving geometry and constraints: frequencies of a two-density inhomogeneous drum. J. Comput. Phys., 171:272-288, 2001.

[PBH04] B. Protas, T-R Bewley, and G. Hagen. A computationnal framework for the regularization of adjoint analysis in multiscale pde systems. J. Comput. Phys., 195:49-89, 2004.

[RC90] B. Rousselet and D. Chenais. Continuité et différentiabilité d'élements propres: Application à l'optimisation de structures. Appl. Math. and Optim., 22:27-59, 1990.

[SHSP89] J. Sanchez-Hubert and E. Sanchez-Palencia. Vibration and coupling of continuous systems. Asymptotic methods. Springer-Verlag, Berlin, 1989.

[SJP92] J. Sokolowski and J-P.Zolesio. Introduction to shape optimization: shape sensitivity analysis, Springer Series in Computational Mathematics, volume 16. Springer-Verlag, Berlin, 1992. 
[SW00] J-A. Sethian and A. Wiegmann. Structural boundary design via level-set and immersed interface methods. J. Comput. Phys., 163:489-528, 2000.

[SZ01] J. Sokolowski and A. Zochowski. Topological derivatives of shape functionals for elasticity systems. Mech. Structures Mach., 29(3):331-349, 2001.

[VB96] L. Vandenberghe and S. Boyd. Semidefinite programming. SIAM review, 38:49-95, 1996.

[WWG03] M-Y. Wang, X. Wang, and D. Guo. A level-set method for structural topology optimization. Comput. Methods Appl. Mech. Engrg., 192:227-246, 2003. 